\title{
Reliable Fault-Tolerant Model Predictive Control of Drinking Water Transport Networks
}

\author{
Deneb Robles ${ }^{\mathrm{a}}$, Vicenç Puig ${ }^{\mathrm{b}}$, Carlos Ocampo-Martinez ${ }^{\mathrm{b}}$, Luis E. Garza ${ }^{\mathrm{a}}$ \\ ${ }^{a}$ Tecnológico de Monterrey, Campus Monterrey, Av. Eugenio Garza Sada Sur No. 2501 Monterrey, N.L. \\ 64489 México \\ ${ }^{b}$ Automatic Control Department, Universitat Politècnica de Catalunya (UPC), Institut de Robòtica $i$ \\ Informàtica Industrial (CSIC-UPC), Llorens i Artigas, 4-6, 08028 Barcelona, Spain
}

\begin{abstract}
This paper proposes a reliable fault-tolerant model predictive control applied to drinking water transport networks. After a fault has occurred, the predictive controller should be redesigned to cope with the fault effect. Before starting to apply the faulttolerant control strategy, it should be evaluated whether the predictive controller will be able to continue operating after the fault appearance. This is done by means of a structural analysis to determine loss of controllability after the fault complemented with feasibility analysis of the optimization problem related to the predictive controller design, so as to consider the fault effect in actuator constraints. Moreover, by evaluating the admissibility of the different actuator-fault configurations, critical actuators regarding fault tolerance can be identified considering structural, feasibility, performance and reliability analyses. On the other hand, the proposed approach allows a degradation analysis of the system to be performed. As a result of these analyses, the predictive controller design can be modified by adapting constraints such that the best achievable performance with some pre-established level of reliability will be achieved. The proposed approach is tested on the Barcelona drinking water transport network.
\end{abstract}

Keywords: Fault tolerance evaluation, model predictive control, actuator-fault configurations, structural analysis, reliability, drinking water transport networks

\section{Introduction}

Potable water is provided to consumers and industry by means of drinking water networks, which are large-scale systems that can be structurally organized in several layers (Ocampo-Martinez et al., 2013):

- A supply layer, composed of water sources, large reservoirs and natural aquifers.

- A transportation layer, linking water treatment and desalination plants with reservoirs distributed all over a city.

\footnotetext{
${ }^{*}$ Corresponding author. Fax: +34 934015750 .

Email address: vicenc.puig@upc.edu (Vicenç Puig)
} 
- A distribution layer, used to meet consumer demands and link reservoirs with consumers.

This paper is focused on the transportation layer and, in particular, on drinking water transport networks (DWTNs). These networks require sophisticated supervisorycontrol strategies to ensure and maintain optimal performance even in faulty conditions. In order to take advantage of these expensive infrastructures, also necessary is a highly sophisticated real-time control (RTC) scheme to ensure optimal performance (Brdys \& Ulanicki, 1994; Ocampo-Martinez et al., 2013). The RTC scheme in a DWTN might be local or global. When control is local, regulation devices only use measurements taken at specific locations. While this control structure is applicable in many simple cases, it may not be the most efficient option for large systems with a highly interconnected and complex sensor and actuator infrastructure. A global control strategy, in contrast, which computes control actions taking into account real-time measurements all through the network, is likely the best way to use infrastructure capacity and all available sensor information. Global RTC deals with the problem of generating control strategies (ahead of time), based on a predictive dynamic model and telemetry readings of the network to optimize operation (Ocampo-Martinez et al., 2013). The multi-variable and largescale nature of DWTNs have led to the use of some variants of model predictive control (MPC) as a global control strategy (Pascual et al., 2013).

Global RTC of DWTNs needs to be operative even in faulty conditions. This problem calls for the use of fault-tolerant control (FTC) mechanisms after a fault is diagnosed so as to avoid the global RTC stopping every time a fault appears. FTC was developed in order to address the growing demand for plant availability (Blanke et al., 2016). The aim of FTC is to keep a plant fully operative by designing its control system such that system performance can be kept close to desirable levels and stability conditions can be maintained, not only when the system is in nominal conditions but also in the presence of system component faults; FTC should, at the very least, ensure acceptable degraded performance (Noura et al., 2009). Tolerance against faults can be embedded in MPC relatively easily in several different ways, as discussed in Maciejowski (2002):

- Changing the constraints in order to represent the fault effect, with the algorithms for actuator faults being especially easy to adapt.

- Modifying the internal plant model used by the MPC in order to reflect fault influence on the plant.

- Relaxing the nominal control objectives in order to reflect system limitations under faulty conditions.

Reviewing the literature, the inclusion of fault tolerance in MPC has already been considered by several authors, including (Zhang \& Jiang, 2008), who provides a detailed review of the state-of-the-art in FTC. Camacho et al. (2010) provides a general overview on how fault tolerance can be embedded in MPC. The inclusion of faulttolerance in MPC has mainly been addressed by considering practical strategies according to the application domain. For example, Prodan et al. (2015) described a 
method for including fault tolerance in MPC for smart grids in order to ensure the proper amount of energy in storage devices and reliable coverage of essential consumer demand. Ocampo-Martinez \& Puig (2009) applied fault tolerance in MPC to sewage networks considering a hybrid systems framework. Yang \& Maciejowski (2012) designed a group of predictive controllers to compensate for the fault effects for each component in a wind turbine. More theoretical aspects have also begun to be studied, such as coupling with active fault diagnosis (Raimondo et al., 2013) and the use of setinvariance theory (Yetendje et al., 2012). More recent additional objectives for MPC controllers, proposed in Sanchez et al. (2015) and Salazar et al. (2015), have been to preserve system health and reliability, respectively.

The research presented in this paper is based on three concepts:

- How fault accommodation/reconfiguration strategies were applied in a linear quadratic regulator (LQR) (Staroswiecki \& Berdjag, 2010).

- The idea that fault configurations should be evaluated before applying FTC strategies (Staroswiecki et al., 2012).

- The idea of using reliability with the FTC design (Guenab et al., 2011).

Starting from these key ideas, we propose a new reliable fault-tolerant MPC scheme for application to DWTNs. After a fault has occurred, the MPC controller should be redesigned to cope with the fault by considering either a reconfiguration or an accommodation strategy, depending on knowledge available on the fault. Before starting to apply the FTC strategy, whether the MPC controller will be able to continue operating after the fault appears should be evaluated. This is done in two ways: first, a structural analysis is done to determine the level of loss in post-fault controllability; second, a feasibility analysis is done of the optimization problem related to the MPC design so as to consider the fault effect on actuator constraints. By evaluating the admissibility of different actuator-fault configurations (AFCs), critical actuators regarding fault tolerance can be identified considering structural, feasibility, performance and reliability analyses. This has been studied in Robles et al. (2012), where only some of the analyses proposed here were considered.

Our approach allows a degradation analysis of the system to be performed in terms of performance and reliability. As a result of this analysis, the MPC controller design can be modified, adapting the constraints so as to achieve the best achievable performance with some pre-established level of reliability. The proposed approach was tested in the Barcelona DWTN, in an application that also shows that relevant information about critical actuators can be extracted by considering the different analyses we propose.

The main contribution of this paper is the design of methodologies for the analysis of the influence of faults taking into account reliability features. As discussed, some of the proposed methodologies have been previously documented but not their application in the considered fault tolerance framework, to the best of our knowledge, after a thorough literature review (a secondary contribution of the paper).

The remainder of the paper is organized as follows. MPC controller design for DTWNs to include fault tolerance is introduced in Section 2. Section 3 describes the 
proposed fault tolerance evaluation approach for the MPC controller after fault occurrence. Section 4 deeply describes the design of the MPC controller such as the reliability can be preserved. The results of an application of this approach to the Barcelona DWTN are provided in Section 5. Finally, Section 6 concludes with some suggestions for further research.

\section{MPC of DWTN with fault-tolerant capabilities}

\subsection{Flow-based control-oriented model}

This paper considers a general DWTN as represented by a digraph $G(\mathcal{V}, \mathcal{E})$ (see Šiljak (1991) for more details), where a set of elements, i.e., $n_{s}$ sources, $n_{x}$ storage elements, $n_{q}$ intersection nodes, and $n_{d}$ sinks, are represented by $v \in \mathcal{V}$ vertices connected by $a \in \mathcal{E}$ links. Due to the network function, water is transported along the links by $n_{u}$ flow actuators (i.e., pipes and valves), passing through reservoirs or tanks, from specific origin locations to specific destination locations. The network is subject to several capacity and operational constraints, and to measured stochastic flows to customer sinks as driven by water demand.

Selecting the volume in storage elements as the state variable $x \in \mathbb{R}^{n_{x}}$, the flow through the actuators as the manipulated inputs $u \in \mathbb{R}^{n_{u}}$, and the demanded flow as additive measured disturbances $d \in \mathbb{R}^{n_{d}}$, the control-oriented model of the DWTN may be described by the following set of linear (or linearized) discrete-time differencealgebraic equations (DAE) for all time instants $k \in \mathbb{N}$ :

$$
\begin{aligned}
x_{k+1} & =A x_{k}+B u_{k}+B_{d} d_{k} \\
0 & =E_{u} u_{k}+E_{d} d_{k}
\end{aligned}
$$

where the difference equation in (1a) describes the dynamics of the storage tanks, and the algebraic equation in (1b) describes static relations in the network (i.e., mass balance at junction nodes). Moreover, $A, B, B_{d}, E_{u}$ and $E_{d}$ are time-invariant matrices of suitable dimensions as dictated by the network topology.

System (1) is subject to hard state and input polytopic constraints given by:

$$
\begin{aligned}
& \mathbb{U} \triangleq\left\{u \in \mathbb{R}^{n_{u}} \mid u_{\min } \leq u \leq u_{\max }\right\} \\
& \mathbb{X} \triangleq\left\{x \in \mathbb{R}^{n_{x}} \mid x_{\min } \leq x \leq x_{\max }\right\}
\end{aligned}
$$

where $u_{\min }, u_{\max }, x_{\min }$ and $x_{\max }$ are the actuator and tank operational limits.

\subsection{Statement of the control problem}

The DWTN (1) is controlled using an MPC law that aims to minimize the operational costs of the DWTN as proposed in economic model predictive control (EMPC) (Rawlings et al., 2012; Limon et al., 2014; Ellis et al., 2014). According to Blanke et al. (2016), the solution of a control problem consists of finding a control law from a given set of control laws $\mathcal{U}$, such that the controlled system achieves the control objectives $\mathcal{O}$ while its behaviour satisfies a set of constraints $\mathcal{C}$. Thus, the solution to the problem is 
completely defined by the triplet $\langle\mathcal{O}, \mathcal{C}, \mathcal{U}\rangle$. In the case of an MPC, the triplet $\langle\mathcal{O}, \mathcal{C}, \mathcal{U}\rangle$ is defined by

$$
\mathcal{O}: \quad \min _{\tilde{x}, \tilde{u}} J(\tilde{x}, \tilde{u}),
$$

subject to:

$$
\begin{array}{ll}
\mathcal{C}: \quad & x_{i+1 \mid k}=A x_{i \mid k}+B u_{i \mid k}+B_{d} d_{i \mid k}, \quad i \in[0, N-1] \subset \mathbb{N}, \\
& 0=E_{u} u_{i \mid k}+E_{d} d_{i \mid k}, \quad i \in[0, N] \subset \mathbb{N}, \\
& u_{i \mid k} \in \mathbb{U}, \quad i \in[0, N-1] \subset \mathbb{N}, \\
& x_{i \mid k} \in \mathbb{X}, \quad i \in[0, N] \subset \mathbb{N}
\end{array}
$$

where

$$
\begin{aligned}
& \tilde{x}=\left(x_{1 \mid k}, \ldots, x_{N \mid k}\right), \\
& \tilde{u}=\left(u_{0 \mid k}, u_{1 \mid k}, \ldots, u_{N-1 \mid k}\right), \\
& \tilde{d}=\left(d_{0 \mid k}, d_{1 \mid k}, \ldots, d_{N-1 \mid k}\right)
\end{aligned}
$$

are the state, input and disturbance sequences over $N$, respectively. $N$ denotes the prediction horizon used by the MPC controller. The sequence $\tilde{d}$ comes from a forecasting module based on existing time-series techniques (see Pascual et al. (2013) and Wang et al. (2015) for more details).

The MPC law belongs to the set $\mathcal{U}$ and is obtained using the receding horizon philosophy (Maciejowski, 2002; Rawlings et al., 2012). This technique consists of solving the optimization problem (3a) from the current time instant $k$ to $k+N$ using $x_{0 \mid k}$ as the initial condition obtained from measurements (or state estimation) at time $k$. Only the first value $u_{0 \mid k}^{*}$ from the optimal input sequence $\tilde{u}^{*}$ (which arises from the solution of the optimization problem (3a)) is applied to the system. At time $k+1$, in order to compute $u_{0 \mid k+1}^{*}$ the optimization problem (3a) is solved again from $k+1$ to $k+1+N$ (i.e., the time window is shifted), updating initial states $x_{0 \mid k+1}$ from measurements (or state estimation) at time $k+1$. The same procedure is repeated for the following time instants.

The objective function $J$ in (3a) collects all the control objectives of the closedloop system, taking the name multiobjective cost function. In general form, (3a) can be written as:

$$
J(\tilde{x}, \tilde{u})=\sum_{i=0}^{n_{J}} \sum_{k=0}^{N} J_{i, k},
$$

where $n_{J}$ is the number of objectives and $J_{i, k}$ corresponds to the evaluation of each particular objective $i$ at time $k$. In the case of DWTNs, (5) typically includes the following objectives (Ocampo-Martinez et al., 2013):

- Minimization of water production and transport costs. The main economic costs associated with drinking-water production are treatment processes, water acquisition and use costs and, most importantly, electricity costs, as delivering drink- 
ing water at appropriate pressure levels through the network implies significant costs in booster pumping and elevation from underground storage. This objective can be mathematically formulated as

$$
J_{e, k} \triangleq \alpha_{k}^{\top} W_{e} u_{k}
$$

where $\alpha_{k} \triangleq\left(\alpha_{1}+\alpha_{2, k}\right) \in \mathbb{R}^{n_{u}}$, which takes into account a fixed waterproduction cost $\alpha_{1} \in \mathbb{R}^{n_{u}}$ and a time-varying water-pumping cost $\alpha_{2} \in \mathbb{R}^{n_{u}}$ that changes in each time instant $k$ according to the dynamic electricity tariff. $W_{e}$ allows the priority economic objective to be weighted in the objective function (5).

- Appropriate safety management of stored water. Water demand must be fulfilled at all times. However, some risk prevention mechanisms need to be introduced in tank management so that the stored volume is maintained above a certain safety value for possible emergencies and to guarantee future water availability. This objective can be mathematically formulated as:

$$
J_{s, k} \triangleq \xi_{k}^{\top} W_{s} \xi_{k},
$$

which penalizes the volume falling below the threshold $s$ by including the following soft constraint

$$
x_{k} \geq s-\xi_{k} \geq 0, \quad \forall k,
$$

where $s \in \mathbb{R}_{+}^{n_{x}}$ is a positive vector of base stocks (the minimum volume in each tank to avoid stock-outs) and $\xi_{k} \in \mathbb{R}_{+}^{n_{x}}$ is a vector of positive slack variables to be minimized, representing the water volume allowed to fall below the desired base stock level. $W_{s}$ allows the priority economic objective to be weighted in the objective function (5).

- Smoothing of control actions. Valves must also operate smoothly in order to avoid major pressure transients in pressurized pipes, as this could result in damage to pipes. The use of a smooth reference changes also helps the lower-level regulator performance. Similarly, water flows requested from treatment plants must have a smooth profile due to operational constraints. To ensure the smoothing effect, control signal variation between consecutive time instants is penalized. This objective can be mathematically formulated as:

$$
J_{\Delta u, k} \triangleq \Delta u_{k}^{\top} W_{\Delta u} \Delta u_{k},
$$

which penalizes control signal variations $\Delta u_{k} \triangleq u_{k}-u_{k-1}$ so as to extend the life of actuators and ensure smooth operation. $W_{\Delta u}$ allows the priority economic objective to be weighted in the objective function (5).

\subsection{Inclusion of fault-tolerant capabilities}

The control problem $\langle\mathcal{O}, \mathcal{C}, \mathcal{U}\rangle$ described in Section 2.2 will now be reformulated to consider faults. If an active FTC strategy is considered, there are two main ways to adapt the MPC law to introduce fault tolerance (Blanke et al., 2016): 
1. System reconfiguration. This consists of finding a new set of constraints $\mathcal{C}_{f}\left(\Theta_{f}\right)$, where $\Theta_{f}$ is the set of parameters changed by the faults such that the control problem $\left\langle\mathcal{O}, \mathcal{C}_{f}\left(\Theta_{f}\right), \mathcal{U}_{f}\right\rangle$ can be solved. This strategy can be applied when the fault detection and isolation (FDI) module does not provide a fault estimation. The faulty components are therefore unplugged by the supervisory system and the control objectives are achieved using non-faulty components. In the case of the actuators, this implies that the model (1) used by the MPC controller is modified as follows:

$$
\begin{aligned}
x_{k+1} & =A x_{k}+\sum_{i \in I_{N}} B_{i} u_{k, i}+B_{d} d_{k}, \\
0 & =\sum_{i \in I_{N}} E_{u, i} u_{k, i}+E_{d} d_{k},
\end{aligned}
$$

where $I_{N}$ is the subset of non-faulty actuators.

2. Fault accommodation. This consists of solving the control problem $\left\langle\mathcal{O}, \hat{\mathcal{C}}_{f}\left(\hat{\Theta}_{f}\right), \hat{\mathcal{U}}_{f}\right\rangle$, where $\hat{\mathcal{C}}_{f}\left(\hat{\Theta}_{f}\right)$ is an estimate of current system constraints and parameters provided by the FDI module. This strategy can be applied when a change occurs in either system structure or parameters. In this strategy, the control law is modified while the remaining elements within the control loop are kept unchanged. In the case of the actuators, this requires that the system model (1) used by the MPC controller should be modified as follows:

$$
\begin{aligned}
x_{k+1} & =A x_{k}+\sum_{i \in I_{N}} B_{i} u_{k, i}+\sum_{i \in I_{F}} \beta_{i}\left(u_{k, i}, \theta_{i}\right)+B_{d} d_{k}, \\
0 & =\sum_{i \in I_{N}} E_{u, i} u_{k, i}+\sum_{i \in I_{F}} \varepsilon_{i}\left(u_{k, i}, \theta_{i}\right)+E_{d} d_{k},
\end{aligned}
$$

where the functions $\beta_{i}$ and $\varepsilon_{i}$ and the parameters $\theta_{i}$ should be estimated by the FDI module for actuators belonging to the faulty actuator subset $I_{F}$.

Note that, in changing the model (1) of the MPC controller using either of the two previous strategies, the controller will consider the effect of the fault in the system model when computing the control action $u_{0 \mid k}^{*}$. According to Maciejowski (2002), this is different from other control laws (e.g., LQR, pole placement), where the control law should be designed off-line for the considered set of faults, so as to produce a bank of controllers that should be gain-scheduled on-line according to the fault features. However, depending on how critical the fault is, the MPC controller will not be able to compute a control input or else the computed control input will not lead to acceptable performance. For this reason, when using an MPC controller the effect of the fault and the admissibility of the obtained control input needs to be evaluated.

\section{Actuator fault-tolerance evaluation}

This section describes a series of analyses to assess the fault-tolerance capabilities of the system after a fault has occurred and before applying a reconfiguration or accommodation strategy to achieve fault tolerance. 
In case that a fault occurs, then:

- The system might have lost some of the properties required to proceed with system control, or

- That system performance is degraded to an unacceptable level and it is not worth continuing with system control by activating fault-tolerant strategies.

\subsection{Admissibility analysis algorithms}

Before starting to apply the FTC strategies described above, it should be evaluated whether the MPC controller will be able to continue operating after fault occurrence. This is done by means of a set of admissibility analysis algorithms, which are based on a structural analysis to determine the loss of post-fault controllability, complemented by a feasibility analysis of the optimization problem related to the MPC design so as to consider the effect of the fault on actuator constraints. Moreover, by evaluating the admissibility of the different AFCs, critical actuators regarding fault tolerance can be identified considering structural, feasibility, performance and reliability analyses.

Let $I$ be the set of system actuators. The different admissibility analysis algorithms consider that the set of all subsets of system actuators is denoted by $2^{I}$. For each subset $K \subseteq I$, corresponding to a given AFC, and using the reconfiguration (or accommodation) approach described in Section 2.3, the algorithms evaluate whether or not a given system property, denoted by $P(K)$, is satisfied (Blanke et al., 2016). Thus,

$$
P_{K}= \begin{cases}1 & \text { if the property is satisfied, } \\ 0 & \text { if the property is not satisfied. }\end{cases}
$$

This evaluation induces the set of all subsets of $I, 2^{I}$, to be partitioned in two classes as follows:

$$
\begin{aligned}
& 2^{I+}=\left\{K \subseteq I ; P_{K}=1\right\}, \\
& 2^{I-}=\left\{K \subset I ; P_{K}=0\right\} .
\end{aligned}
$$

The class $2^{I+}$ contains all the subsets of the actuators for which $P_{K}$ is satisfied. Thus, the admissibility analysis mainly aims to identify the following (see Figure 2):

- Critical actuators, i.e., the set of actuators that are required to satisfy $P_{K}$. For every analysis in Figure 1, a set of critical actuators will be identified.

- Redundant actuators, i.e., the actuators that are not critical for correct functioning of the system. These may be excluded as $P_{K}$ will continue to be satisfied.

- Redundancy degree, consisting of the number of extra non-critical actuators through which $P_{K}$ could hold. There are two types of redundancy: weak (corresponding to the largest number of sequential faults that can be tolerated in the best case scenario, i.e, while continuing to satisfy $P_{K}$ ) and strong (corresponding to the smallest number of sequential faults that can be tolerated in the worst case scenario). 


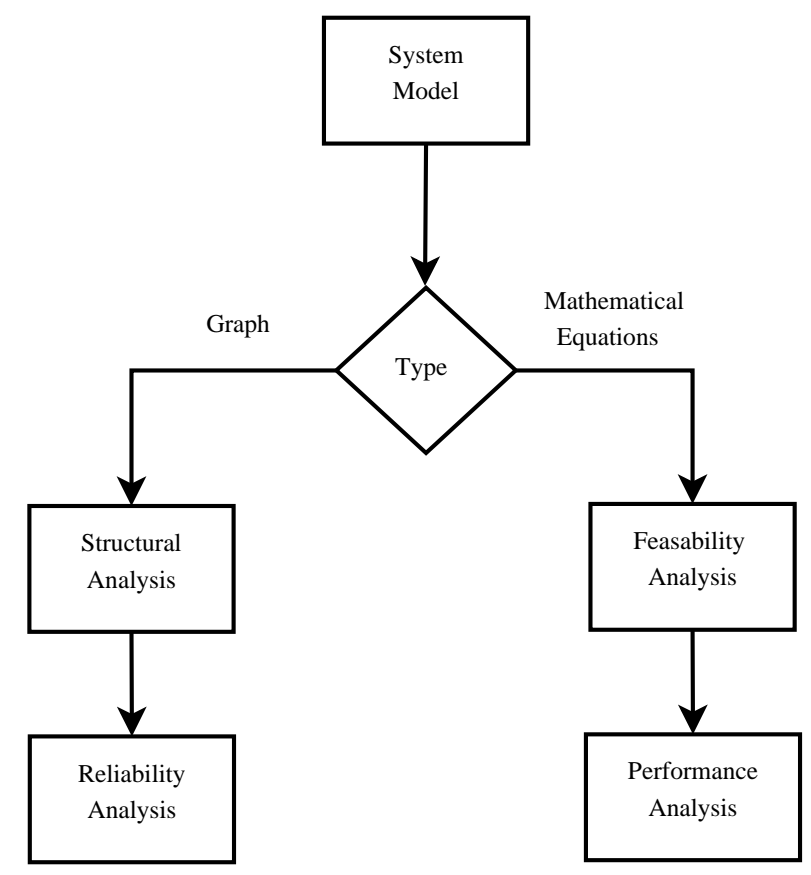

Figure 1: Flow diagram of the proposed actuator fault-tolerance evaluation approach

The approach proposed here consists of a set of analyses based on both the graph and the mathematical model of the system (see Figure 1):

- From the system graph, the structural analysis allows us to determine whether or not the system with a given AFC is structurally controllable. It does this by checking the existence of at least one path linking demands with sources. At this stage, all possible paths linking demands and sources are also determined. Using this information, the reliability of the AFC can also be evaluated.

- From the system mathematical model, a constraint satisfaction problem (CSP) can be formulated that allows a feasibility analysis to be performed. This analysis allows the physical capacity of the system to be checked considering constraints in actuators and states (see (3a)). Moreover, as a complementary analysis, the closed-loop performance based on a given global objective for the AFC can be evaluated.

These two sets of analyses are complementary. When a reconfiguration strategy is used, connectivity between demands and sources may be lost when the faulty actuator is removed (see Section 2.3). This will affect both controllability and reliability. However, those properties do not take into account the physical limitations of the system actuators. Hence, although connectivity is preserved, the MPC-related optimization problem might lead to an unfeasible solution, due either to the lack of capacity of the remaining actuators or the poor performance of the control loop. This happens when 


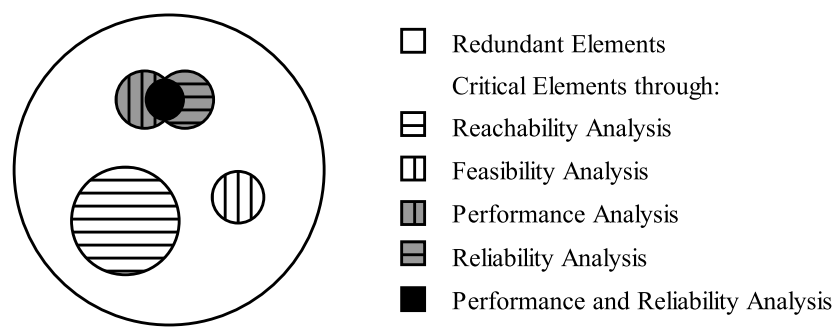

Figure 2: Critical and redundant actuators of the system

an accommodation strategy is used, since although the connectivity among elements is preserved (the faulty actuator is not removed), the resulting MPC-related optimization problem may be unfeasible or the closed-loop control scheme may perform poorly.

As a result of the application of the methodology presented in Figure 1, it is possible to determine critical actuators as follows (type of analysis in brackets):

- Actuators that are essential to preserving demand-source connectivity (by means of structural controllability analysis).

- Actuators that are indispensable to preserving the capacity to move the desired water volume from sources to meet demands taking into account actuator physical constraints (by means of structural controllability analysis).

- Actuators whose malfunction generates high suboptimality of the considered control objective if the system is maintained in operation after fault detection (by means of performance analysis).

- Actuators whose malfunction does not guarantee reliable operation of the system (by means of reliability analysis).

Figure 2 depicts the different types of critical actuators that can be identified applying the sequence of analyses presented in Figure 1. Results for each analysis are considered in subsequent analyses, in such a way that actuators that are considered critical at a given stage of the methodology might not be further considered in later analyses.

\subsection{Analyses based on the system graph}

\subsubsection{Structural analysis algorithm}

The structural analysis algorithm copes with connectivity properties of the system without considering the actual value of the model parameters or the limitations of the actuators ${ }^{1}$. This test is used to evaluate the admissibility of a given AFC when the reconfiguration FTC strategy is used, i.e., when an actuator is removed after fault occurrence and the system is controlled by the remaining actuators.

The algorithm starts by determining the $\operatorname{digraph}^{2} G(\mathcal{V}, \mathcal{E})$ of the model used for

\footnotetext{
${ }^{1}$ See Blanke et al. (2016) for important definitions related to the topic.

${ }^{2}$ See Šiljak (1991) for details on how to obtain a digraph from the system model.
} 


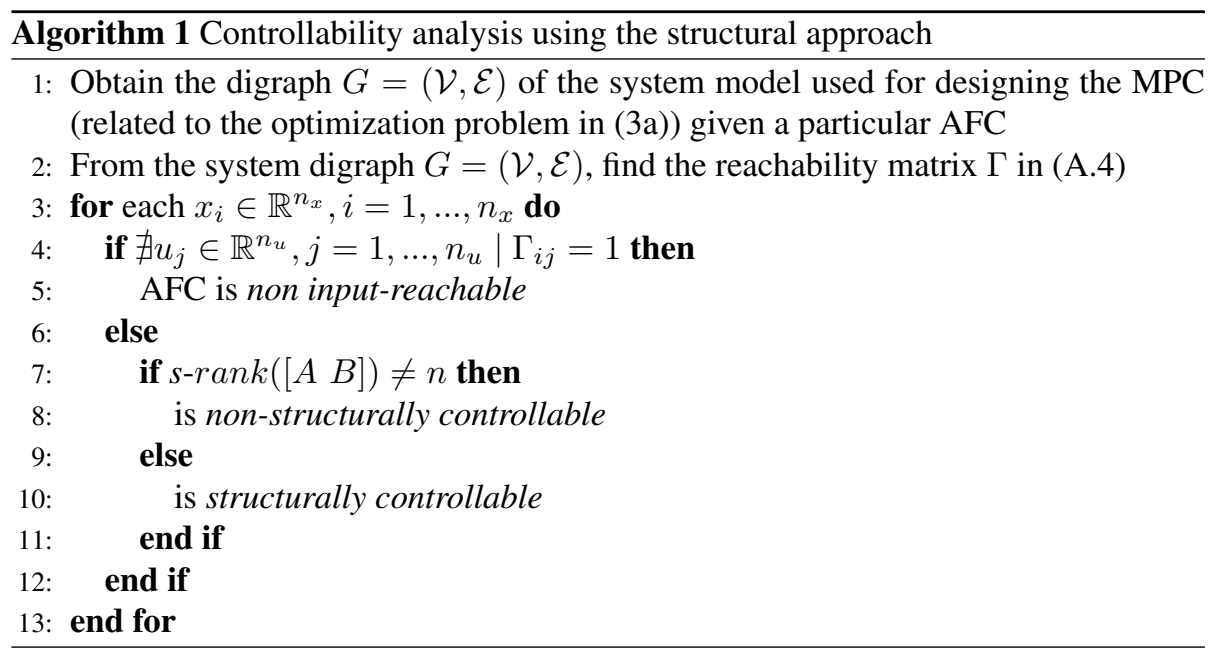

the MPC controller. Using the digraph, the structural controllability of the system for a given AFC will be evaluated. If this property is preserved after the actuator fails, the AFC is admissible, i.e., it is able to tolerate the fault; otherwise, the AFC is not admissible. To evaluate structural controllability from the system graph, some basic graph theory concepts (reviewed in the Appendix) will be used (see (Bondy \& Murty, 1982) for more details). Using Theorems 1 and 2 from the Appendix, Algorithm 1 will perform the structural controllability analysis for a given AFC.

\subsection{Analyses based on the system mathematical model}

\subsubsection{Feasibility analysis algorithm}

To evaluate the admissibility of the control of a given AFC when system constraints (2) are considered, it is not possible to use the structural analysis algorithm ${ }^{3}$. presented in Section 3.2.1.

Feasibility in an MPC controller design is a key property to be satisfied before the control action can be computed by solving the optimization problem (3a) (Maciejowski, 2002). In this case, the admissibility evaluation problem for a given AFC can be naturally handled as a CSP (see the Appendix for a definition). Consequently, the feasibility evaluation of the MPC-related optimization problem (here for a given $\mathrm{AFC}$ using the reconfiguration strategy $)^{4}$ can be checked using Algorithm 2.

\subsubsection{Performance analysis algorithm}

The degradation of the control objective in a fault situation can be quantified by means of maximal loss of efficiency $\rho$ with respect to the objective function in a non-

\footnotetext{
${ }^{3}$ This would also be the case when an accommodation FTC strategy is used, since the actuator would not be removed after the fault but would be operated under the remaining operating range estimated by the FDI module.

${ }^{4}$ In case that an accommodation strategy is used, the faulty model used in Algorithm 2 should be replaced by the one used in (12).
} 


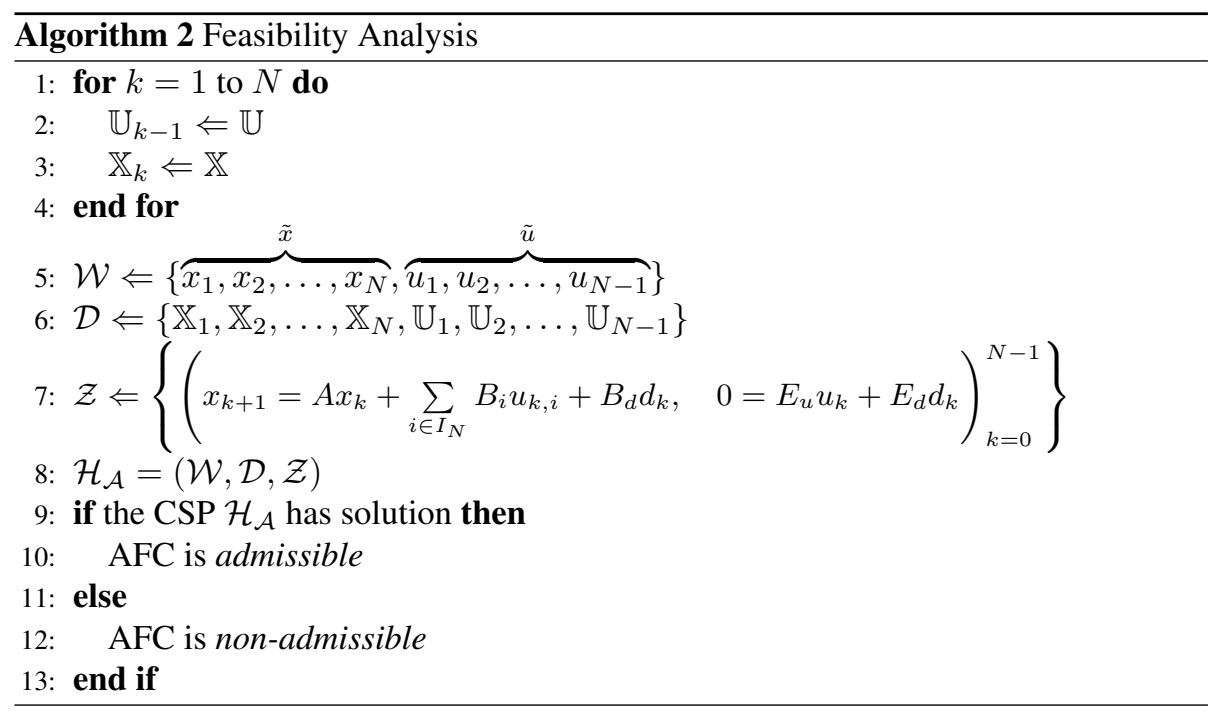

fault situation $J_{0}$. This fact establishes whether of not the control objective degradation after an actuator fault $J_{f}$ is admissible. Thus, an AFC is admissible regarding performance if the following condition is satisfied: $J_{f} \leq(1+\rho) J_{0}$. This condition will enable a performance analysis of the AFC considering the faulty actuator, with either an accommodation or a reconfiguration strategy.

The procedure for evaluating the performance admissibility of the controller with respect to the fault situation is summarized by Algorithm 2, modifying the constraints defined in step 7 to add a new constraint:

$$
\phi_{x_{N}}+\sum_{i=0}^{N-1} \Phi_{i}\left(x_{i}, u_{i}\right) \leq(1+\rho) J_{0} .
$$

Note that, as in the case of the feasibility analysis, the existence of a solution to the CSP associated with MPC performance evaluation for a given AFC using the reconfiguration strategy ${ }^{5}$ can be proved by Algorithm 2 but including the new constraint (17), which considers the admissibility condition with respect to control performance over the prediction horizon $N$ stated in the MPC controller.

\section{MPC redesign to preserve reliability}

\subsection{Reliability analysis algorithm}

Reliability is defined as the probability that a given component (or system) will accomplish its intended function during a given period of time and in specific operating

\footnotetext{
${ }^{5}$ If an accommodation strategy is used, the fault model used in Algorithm 2 should be replaced by the one used in (12).
} 
conditions and environments (Gertsbakh, 2000). In other words, it is the probability of success in accomplishing a task or achieving a desired property in a process, based on proper operation of components. The main advantages of including a reliability analysis are as follows:

- Information on component health is integrated in controller design and improves the life of the system components

- Reliability information on the system can be considered as design criteria to be used in MPC implementation including FTC capabilities

- Essential actuators whose malfunction causes abrupt system reliability decay are identified.

In the case of DWTNs, reliability is understood as the ability of the network to provide an efficient water supply to consumers under both normal and abnormal operating conditions. For this reason, reliability is a measure of DWTN performance. Reliability in DWTNs has already been considered in the literature (Torii \& Lopez, 2012; Ostfeld, 2001).

When a reconfiguration FTC strategy is used, the reliability of DTWNs can be affected due to the probabilities of success of each of the components in the new configuration. For this case, the admissibility evaluation problem of a given AFC can be handled as composite reliability of the subsystems in the system. In particular, since reliability in DTWNs is related to guaranteed supply to consumers, it can be determined based on all the possible paths linking demands and sources from the network graph already obtained in the structural analysis.

The global reliability of a system, denoted by $R_{g, k}$, generally consists of the decomposition of its subsystems into elementary combinations of serial and parallel subsystems that can be extracted from the matrix containing all paths linking demands and sources (Guenab et al., 2011):

- Reliability of $n_{p}$ parallel subsystems is defined as:

$$
R_{p, k}=1-\prod_{i=1}^{n_{p}}\left(1-R_{i, k}\right)
$$

- Reliability of $n_{s}$ serial subsystems is defined as:

$$
R_{s, k}=\prod_{i=1}^{n_{s}} R_{i, k}
$$

where $R_{i, k}$ represents the reliability of the $i$-th actuator (or subsystem) at time $k$ and where $\lambda_{i, k}$ is the failure rate modelled as an exponential distribution:

$$
R_{i, k}=e^{-\lambda_{i, k} k}
$$


Thus, overall system reliability is given by:

$$
R_{g, k}=\prod_{i=1}^{n_{s}}\left(1-\prod_{i=1}^{n_{p}}\left(1-R_{i, k}\right)\right) .
$$

Algorithm 3 shows the reliability evaluation of a given AFC based on computing system reliability. Since the calculation of reliability for each and every AFC could impose a great computational burden, to save time, the path matrix that contains all the possible paths in the system graph (see Appendix) is used. This matrix has the following structure:

\begin{tabular}{c|c|c|c|c|c|} 
& $p_{1}$ & $p_{2}$ & $p_{3}$ & $\ldots$ & $p_{n_{p h}}$ \\
\hline$u_{1}$ & 1 & 0 & 1 & $\ldots$ & 0 \\
$u_{2}$ & 0 & 1 & 1 & $\ldots$ & 1 \\
$u_{3}$ & 1 & 0 & 0 & $\ldots$ & 1 \\
$\vdots$ & $\vdots$ & $\vdots$ & $\vdots$ & $\vdots$ & $\vdots$ \\
$u_{n_{u}}$ & 0 & 1 & 1 & $\ldots$ & 1
\end{tabular}

where $n_{p h}$ is the number of path and 1 and 0 indicate the presence and absence, respectively, of an actuator in the path. Each time a component malfunctions, the row assigned to that actuator is withdrawn along with all the paths that make use of it. To evaluate fault tolerance for the rest of the system, the reliability index $R_{g, k}$ should be greater than a specific admissibility threshold $R_{t h}$ at a given time horizon $k_{e n d}$, both defined by the user.

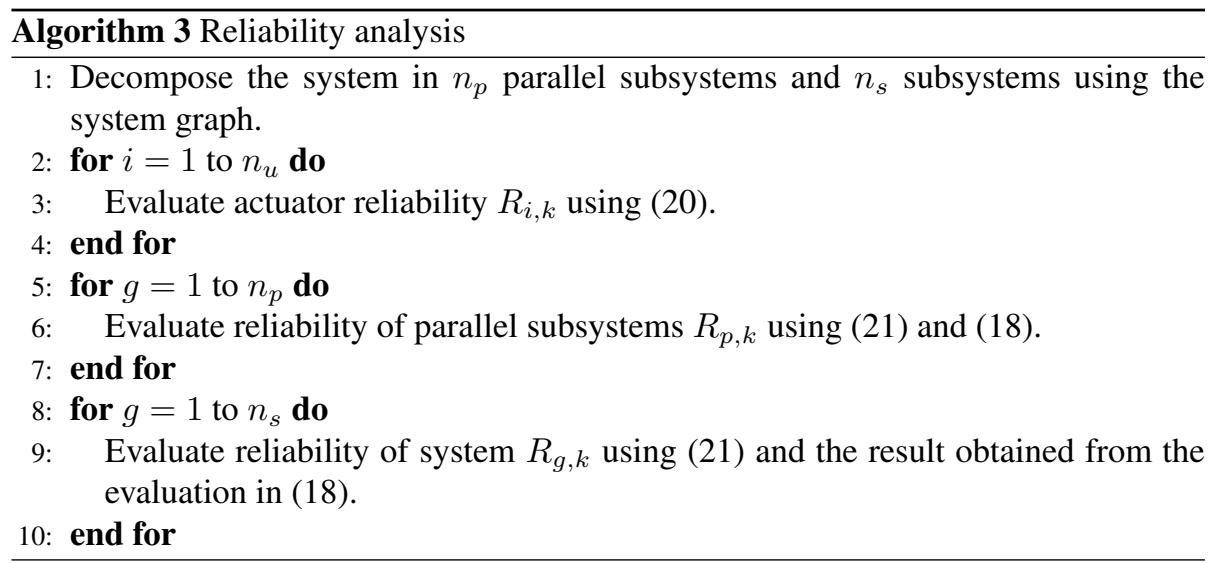

\subsection{MPC redesign to preserve reliability}

When a fault occurs, the MPC law is modified to cope with the fault, as discussed in Section 2.3. As explained in Guenab et al. (2011), the value of the actuator failure rate changes because the control action should be increased in order to compensate for the fault effect. In this case, energy consumption increases and the value of the failure rate also increases due to the actuator load increment. Thus, there is an interplay 
between maintaining closed-loop performance and reliability. To maintain the desired performance, the relationship between the actuator load increment and reliability can be established. One of the most commonly used relationships is based on assuming that the actuator failure rate changes with the load through the following exponential law:

$$
\lambda_{i, k}=\lambda_{i}^{o} e^{\beta_{i} u_{i, k}}
$$

where $\lambda_{i}^{o}$ represents the baseline failure rate (nominal failure rate) and $u_{i}$ is the control action for the $\mathrm{i}-t h$ actuator. Parameter $\beta_{i}$ is a fixed factor that depends on the actuator characteristics. Thus, the reliability of the actuator can be expressed in terms of its load as follows:

$$
R_{i, k}=e^{\lambda_{i} k}=e^{\lambda_{i}^{o} e^{\beta_{i} u_{i, k}} k} .
$$

Consider that a predefined reliability threshold $R_{t h}$ should be maintained until the end of the system mission at time $k_{\text {end }}$. This threshold defines the minimal acceptable reliability value in the degraded fault mode. The aim is to translate this threshold to a load threshold that can be applied to the actuator. This actuator load threshold can be derived from (24) as follows:

$$
\left|u_{i, t h}\right|=\frac{1}{\beta_{i}} \ln \left(\frac{\ln R_{i, t h}}{\lambda_{i}^{o} k_{\text {end }}}\right) .
$$

Hence, the MPC controller ( $3 a$ ) can be redesigned by including the following constraint in the i-th actuator control:

$$
u_{i} \in\left[-u_{i, t h}, u_{i, t h}\right]
$$

However, as discussed in Weber et al. (2012), this will only preserve the reliability of the i-th actuator. In order to preserve the reliability of the whole DWTN, the new actuator constraints (26) should be derived taking into account the reliability expression (21) and the reliability threshold $R_{t h}$ at the end of the MPC prediction horizon $N$. This can be achieved by formulating a CSP problem, such as that reflected in Algorithm 4, which considers, as constraints, the reliability of the DWTN in (21) derived by means of Algorithm 3 in terms of the reliability of each actuator, the impact of actuator load (see (24)) and the actuator operational constraints defined in (3a).

After solving the CSP problem in Algorithm 4, to solve the optimization problem associated the MPC problem, the resulting updated actuator constraints are used instead of the actuator operational constraints defined in (3a). In this way, we can guarantee that the MPC controller computes a control sequence that preserves reliability. There is, of course, a trade-off between reliability and performance. Increasing the reliability threshold $R_{t h}$ will imply a reduction in the DWTN performance but will extend the life of the remaining actuators.

\section{Application to the Barcelona DWTN}

\subsection{Case study description}

The Barcelona DWTN is used as the case study of this paper. This network is managed by Aguas de Barcelona SA (AGBAR), which supplies drinking water to Barcelona and its metropolitan area. The main water sources are the rivers Ter and Llobregat. 


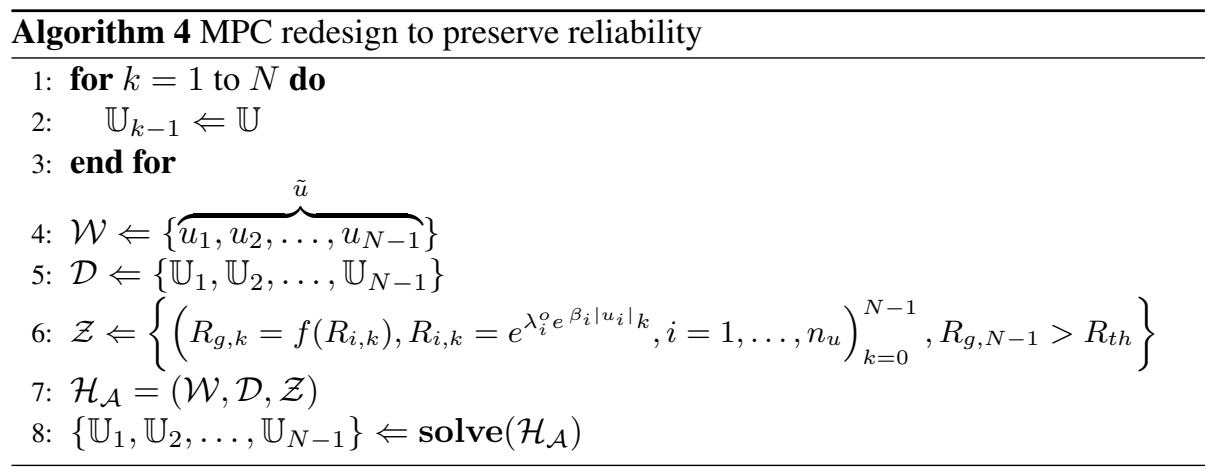

Currently, there are four water treatment plants: the Abrera and Sant Joan Despí plants, which extract water from the Llobregat river; the Cardedeu plant, which extracts water from the Ter river; and the Besòs plant, which treats underground water from the Besòs river aquifer. There are also several underground sources (wells) that can provide water through pumping.

For this case study, ten sources were considered, consisting of seven underground and three surface sources, which currently provide an inflow of about $12 \mathrm{~m}^{3} / \mathrm{s}$. The Barcelona DWTN is comprised of 63 tanks and 130 actuators distributed across 46 valves and 84 pumps $^{6}$. Figure 3, which shows the general topology of the network, depicts a complex system in terms of its elements and the relationships and connections among them. Figure 4 shows the graph derived from this network; the nodes correspond to reservoirs or pipe merging/splitting nodes and the arcs correspond to actuators (valves and pumps). Five of the pumps are used to draw water from underground sources and the remaining pumps satisfy water demand at appropriate pressure levels. The network has 88 main water consumption sectors (for further information regarding the Barcelona DWTN, see (Pascual et al., 2013)). Both the demand episode and the calibration set-up of the network are as established by AGBAR. The AGBAR control centre has a telecontrol system for network management. The Barcelona DWTN also has some 98 remote stations, which manage about 450 elements in real time, including flow meters, pumps, valves and chlorine dosing instruments.

The system control objective set for the MPC controller is to minimize operational costs (water transport and production for the entire network) while satisfying water demand for each consumption sector (Pascual et al., 2013). Thus, recapping on Section 2 , the financial objective in (6) can be written as follows:

$$
J_{k}=\sum_{i=0}^{N-1}\left[\alpha_{1}+\alpha_{2, k}\right] u_{k},
$$

\footnotetext{
${ }^{6}$ For our purposes we refer to pumps, although, in fact, pumping stations would be more correct, as the stations consist of arrays of pumps with a given local control/coordination strategy regarding certain outflow requirements.
} 


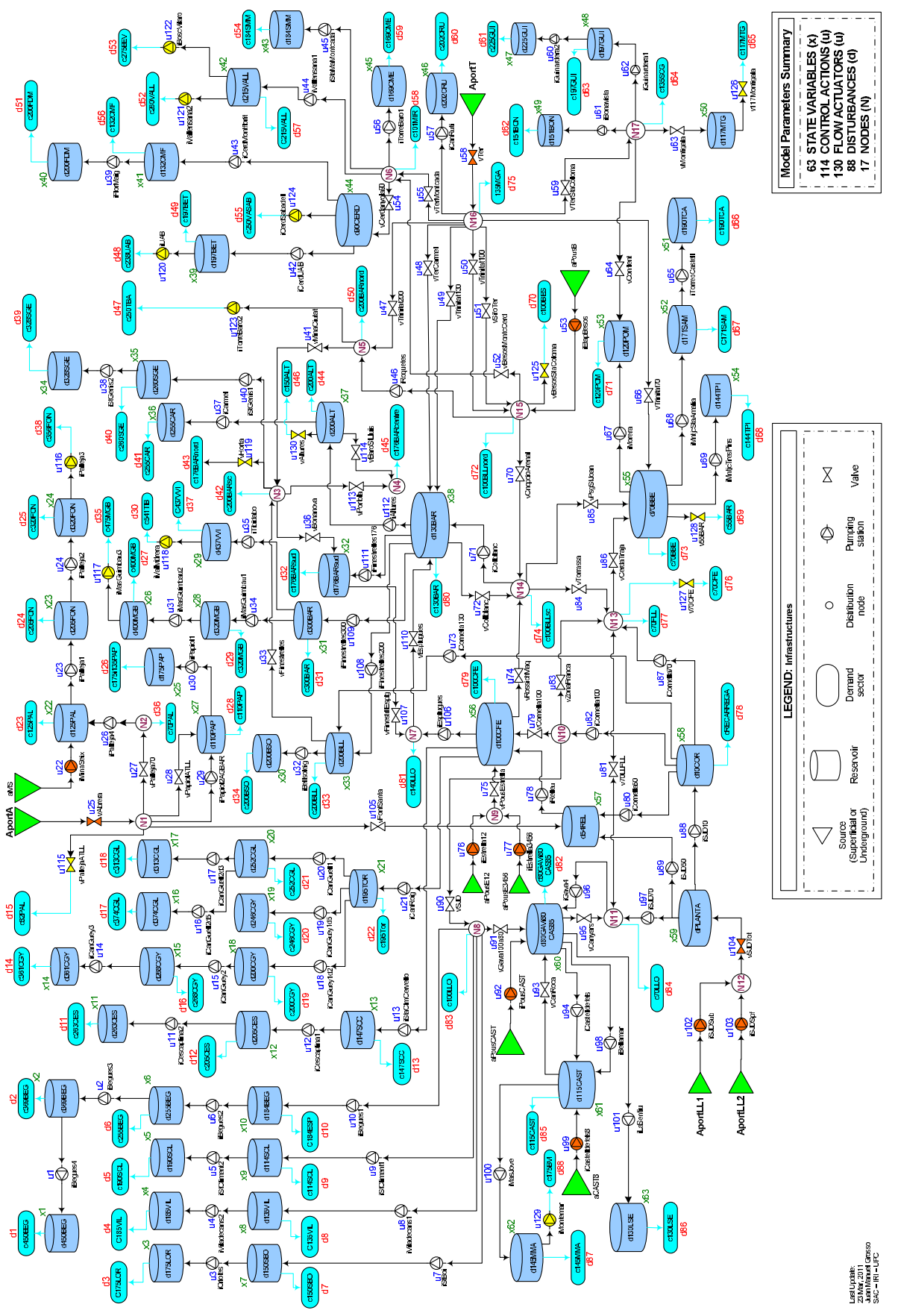

Figure 3: Barcelona Drinking Water Transport Network 


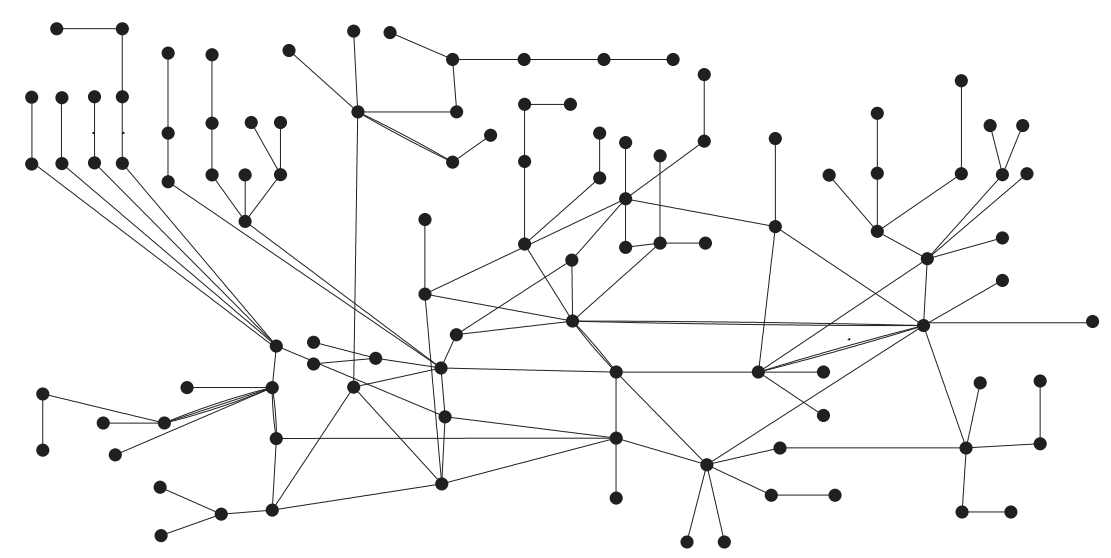

Figure 4: Graph of the Barcelona DWTN

which takes into account the water cost $\alpha_{1}$ (price of water at source) and the electricity cost $\alpha_{2, k}$ (operation of pumps and valves). Note that the time variance of $\alpha_{2}$ is due to the fact that pumping costs vary according to the time of day. The prediction horizon $N$ is 24 hours. No terminal cost is considered in this application.

Demands are imposed as equality constraints in the model (1) used by the MPC controller, which, in the case of the DWTN, can be expressed in discrete-time statespace form (1) using a sampling time $T_{s}=1$ hour. $x \in \mathbb{X} \subseteq \mathbb{R}^{n_{x}}$ is the state vector corresponding to the water volumes of the $n_{x}=63$ tanks, $u \in \mathbb{U} \subseteq \mathbb{R}^{n_{u}}$ represents the vector of manipulated flows through the $n_{u}=130$ actuators (pumps and valves) and $d \in \mathbb{D} \subseteq \mathbb{R}^{p}$ corresponds to the vector of the $p=88$ water demands (consumption sectors).

There are 16 nodes in the Barcelona DWTN and since demand can be forecasted, these are assumed to be known. Thus, $d$ is a known vector of non-negative elements that contains the measured disturbances (demands) affecting the system.

\subsection{Results}

Several tests and analyses were performed for the Barcelona DWTN case study to illustrate the proposed methodology. Figure 1 shows the sequence of tests applied. In this section, all the capabilities of each analysis are explored, while Section 5.3 describes only the ones necessary for this study. The results were obtained using a 1.5 $\mathrm{GHz}$ and $2.00 \mathrm{~Gb}$ RAM Intel(R) Core(TM)2 Duo PC . Matlab ${ }^{\circledR}$ and Tomlab were used to perform the simulations.

The structural analysis was carried out using the computed reachability matrix and path computation, which, as expected, produced equivalent results. However, each technique yielded several additional results that provided important information concerning to the operation and behaviour of the DWTN. From the reachability analysis, we could determine which states were structurally controllable, while the path computation analysis obtained all possible paths from a source to a destination node as well as, for each path, an approximate operational cost (according to the electricity cost of 
each element) and a maximal water flow (according to the physical constraints of the actuators). In this stage, critical actuators were located and different approaches were used according to the applied strategy. Although a fault scenario with a faulty actuator at each time instant was considered in both cases, the representation of the malfunction was denoted in different ways. In the reachability analysis, the malfunction was determined from the state-space matrices (a zero value was forced in the position where a connection value previously existed between the state and the actuator failure). In path computation, all paths with the faulty component were extracted from the path matrix (22). From this study, the critical actuators for each state and for the whole network could be identified. Note that although the results obtained by both techniques in the structural analysis were similar, the computation time required for the reachabilitymatrix-based strategy was much higher, at almost 200 times the time consumed by the path computation technique ( $579 \mathrm{~s}$ vs. $3 \mathrm{~s}$ ).

The feasibility analysis can only be implemented if the previous analysis is first made, since its implementation is based on the path matrix calculation. The result of this analysis was a set of paths that guaranteed that demand was satisfied, taking into account the physical constraints of the network actuators. The cost of maintaining correct network operations was also obtained in this stage. The time consumed by this analysis was $1.57 \mathrm{~s}$.

Performance was computed using the objective function (27) and the actuator constraints. The analyses were performed taking into account faulty components and comparing the corresponding performance with the fully operative case (non-faulty system). The computation time needed for this analysis was $8 \mathrm{~s}$. Finally, the reliability analysis showed the level of reliability of each component and path and of the whole network. AFCs were analysed by extracting all paths using the faulty actuator and re-computing the reliability of the DWTN. Two rankings were computed: the first one according to demand satisfaction, showing which demands were more likely to be unsatisfied; and the second one according to the most critical actuators, showing how the reliability of the entire network decreased if those actuators were damaged. The computation time in this case was $5 \mathrm{~s}$.

\subsection{Discussion}

Although each of the previous analyses can individually provide a great deal of information about the fault tolerance of a network, linking them up reduces the computational burden. In order to clearly present and easily discuss the proposed methodologies, a smaller portion of the Barcelona DWTN (see Figure 5) was used for illustrative purposes.

The first test consisted of locating the critical network actuators by means of a structural analysis. These critical actuators are those without which (outage) path connectivity is lost. The results of this analysis, summarized in Tables 1 and 2, point to an important number of critical actuators within the network, due to the topology and the way of connecting network elements, as most actuators (valves or pumps) are the only link between tanks and demands. Therefore, if an actuator fails, then the corresponding demand will not be satisfied. Note that the information shown in Tables 1 and 2 is particularly significant for AGBAR (the manager of the water infrastructure), since it 


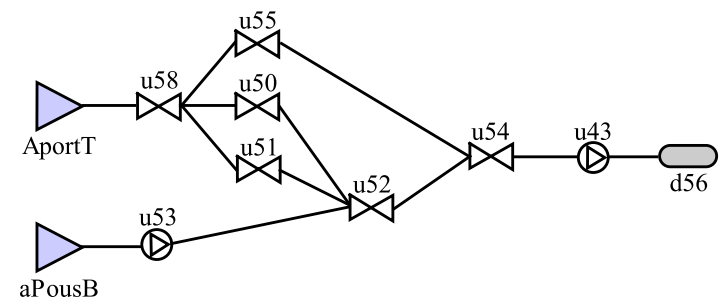

Figure 5: Portion of the DWTN related to Demand 56

Table 1: Structural Critical Actuators (towards tanks)

\begin{tabular}{cccccccc}
\hline No. & Name & No. & Name & No. & Name & No. & Name \\
\hline 122 & iAltures & 15 & iCanGuey2 & 62 & iGuinardera1 & 30 & iPapio11 \\
10 & iBegues1 & 14 & iCanGuey3 & 60 & iGuinardera2 & 88 & iSJD10 \\
6 & iBegues2 & 21 & iCanRoig & 101 & iLaSentiu & 7 & iStBoi \\
2 & iBegues3 & 57 & iCanRuti & 34 & iMasGuimbau1 & 9 & iStCliment1 \\
1 & iBegues4 & 37 & iCarmel & 31 & iMasGuimbau2 & 5 & iStCliment2 \\
32 & iBellsoleig & 43 & iCerdMontflorit & 100 & iMasJove & 40 & iStGenis1 \\
61 & iBonavista & 42 & iCerdUAB & 68 & iMntjcStaAmalia & 38 & iStGenis2 \\
20 & iCanGuel11 & 12 & iCesalpina1 & 69 & iMntjcTresPins & 13 & iStaClmCervello \\
17 & iCanGuel12d3 & 11 & iCesalpina2 & 3 & iOrioles & 45 & iStaMaMontcada \\
16 & iCanGuel12d5 & 82 & iCornella100 & 23 & iPalleja1 & 35 & iTibidabo \\
18 & iCanGuey1d2 & 39 & iFlorMaig & 24 & iPalleja2 & 56 & iTorreBaro1 \\
19 & iCanGuey1d5 & 109 & iFnestrelles300 & 27 & vPalleja70 & 65 & iTorreoCastell \\
44 & iVallensana1 & 8 & iViladecans1 & 4 & iViladecans2 & 25 & vAbrera \\
54 & vCerdanyola90 & 63 & vMontigala & 90 & vSJD & 59 & vTerStaColoma \\
104 & vSJDTot & 58 & vTer & & & & \\
\hline
\end{tabular}

identifies the critical elements in the network for surveillance/correction policies to be implemented in the event of element damage (fault).

Applying the first test to the network, as depicted in Figure 5, four possible paths were detected. These were:

$$
\begin{aligned}
& \text { Path 1: Aport } T \rightarrow u 58 \rightarrow u 50 \rightarrow u 52 \rightarrow u 54 \rightarrow u 43 \rightarrow d 56 \\
& \text { Path 2: AportT } \rightarrow u 58 \rightarrow u 51 \rightarrow u 52 \rightarrow u 54 \rightarrow u 43 \rightarrow d 56 \\
& \text { Path 3: AportT } \rightarrow u 58 \rightarrow u 55 \rightarrow u 54 \rightarrow u 43 \rightarrow d 56 \\
& \text { Path 4: aPousB } \rightarrow u 53 \rightarrow u 52 \rightarrow u 54 \rightarrow u 43 \rightarrow d 56
\end{aligned}
$$

Analysing the structure of the network, as depicted in Figure 5, it can be observed that it contains two critical actuators: 54 and 43. If either of these actuators fail, then Demand 56 will not be satisfied. All the remaining actuators can be considered as 
Table 2: Structural Critical Actuators (towards demands)

\begin{tabular}{cccccccc}
\hline No. & Name & No. & Name & No. & Name & No. & Name \\
\hline 115 & vPallejaATLL & 116 & iPalleja3 & 117 & iMasGuimbau3 & 118 & iVallvidrera \\
119 & vHorta & 120 & iUAB & 121 & iVallensana2 & 122 & iBoscVilaro \\
123 & iTorreBaro2 & 124 & iCerdSabadell & 125 & vBesosStaColoma & 126 & v117Montigala \\
127 & v70CFE & 128 & v55BAR & 129 & iMontemar & 130 & vAltures \\
\hline
\end{tabular}

redundant actuators.

The second analysis done to the Barcelona DWTN was to identify the actuators whose physical constraints limit water transport capacity through a certain path. Note that this analysis did not consider any fault in those actuators. The analysis, performed using Algorithm 2, also pinpointed several alternative paths through which water transport is possible (or even mandatory) given the constraints of the paths for supplying demands.

Results for this last analysis considering the whole DWTN identified other critical actuators: 26, 52 and 91 (namely iPalleja4, vBesosMontCerd and vGavalo0a80, respectively, in Figure 3). Note that the increase in the number of critical actuators, taking into account their physical constraints, is not significant. For the network in Figure 5, actuator 52 is not a critical element according to the structural controllability property, meaning that connectivity is not lost when this component fails. However, the feasibility analysis determined that this actuator was in fact critical when the actuator physical constraints were considered. Actuator 52 cooperates with a flow of water to satisfy the demand that cannot be satisfied with a flow through a single path.

The third analysis identified the optimal paths to reach a selected destination node without considering the system constraints, i.e., the structural optimal paths. This analysis was performed using the structural algorithm, as explained in Section 3.2.1. For the smaller network the cost of each path was computed, corresponding to the electricity cost of the actuators for both paths and the cost of water treatment in a determined source. For paths 1, 2 and 3, the cost was 0.54 e.u. ${ }^{7}$, while for path 4 the cost was 0.77 e.u. This small example would indicate that any of the first three paths is optimal for satisfying Demand 56.

A criterion to decide which of the three paths is optimal for this demand is to calculate the maximum flow of water for each path, which can also be computed in this analysis and is given by the smallest value of the maximum flow of water of the actuators in a given path. In this case, since all paths were restricted to $0.3 \mathrm{~m}^{3} / \mathrm{s}$, due to the physical capacities of actuator 43, any of the first three paths are recommended. However, if actuator 43 were not considered, path 1 would be the optimal path as it has a maximum flow of $2.2 \mathrm{~m}^{3} / \mathrm{s}$, while in the other paths, actuator 55 is restricted to $0.35 \mathrm{~m}^{3} / \mathrm{s}$, and actuators 51 and 52 to $0.8 \mathrm{~m}^{3} / \mathrm{s}$.

The fourth analysis consisted of identifying the set of optimal paths including the

\footnotetext{
${ }^{7}$ Note that costs are given in economic units (e.u.) rather than real units $(€)$ for confidentiality reasons.
} 
Table 3: Entire DWTN Performance Analysis

\begin{tabular}{ccc}
\hline Actuator No. & Faulty cost [e.u.] & Cost overrun [\%] \\
\hline 41 & 514.44 & 2.43 \\
47 & 515.94 & 2.73 \\
74 & 528.05 & 5.14 \\
78 & 557.62 & 11.03 \\
86 & 515.08 & 2.55 \\
89 & 556.22 & 10.74 \\
97 & 510.49 & 1.64 \\
102 & 539.87 & 7.49 \\
103 & 552.21 & 9.95 \\
\hline
\end{tabular}

objective function (27) and the system constraints (2a)-(2b). Path details are not provided here, but the total costs of maintaining the whole DTWN in proper working order and satisfying all its demands was 502.25 e.u. In the case of the network depicted in Figure 5, the optimal path obtained from the fourth analysis was path 4. Although it may appear that, when only Demand 56 is considered without the interconnection of the entire network, the other paths are less costly when the entire network is considered, this is not true. The actuators used in path 4 are also used to satisfy other demands, so sharing components results in an optimal solution.

The fifth test was performance analysis, taking into account the critical actuators already identified in the previous tests, with the difference in costs showing the impact that a single faulty actuator could have on an entire network. Results from this analysis are summarized in Table 3. Note that all comparisons took into account an optimal functioning cost (under non-faulty conditions) of 502.25 e.u. Moreover, fault cost denotes the functioning cost under faulty conditions.

According to the analysis of the entire DWTN, some actuators did not have a significant impact on the total performing cost (e.g., actuators $28,29,33,64,71,80,81$, $85,87,94,107,108,113$ ). However, other actuators (such as 78 or 89 ) significantly increased cost, taking into account daily estimates. These latter actuators are shown in Table 3. Degradation in costs obtained with this analysis can be the foundation for the introduction of redundant actuators in the network or an alternative fault tolerance strategy. For the network depicted in Figure 5, the performance analysis shows that the cost of maintaining operations for the network with a fault in any of these actuators does not increase the cost.

The accommodation and reconfiguration strategies presented in Section 2.3 are now illustrated for the case of a fault in actuator 108 (named vTerMontcada), which according to the previous analysis, is redundant. First the reconfiguration strategy is illustrated. Figure 6 presents the volume behaviour of tank 33, which is supplied by 


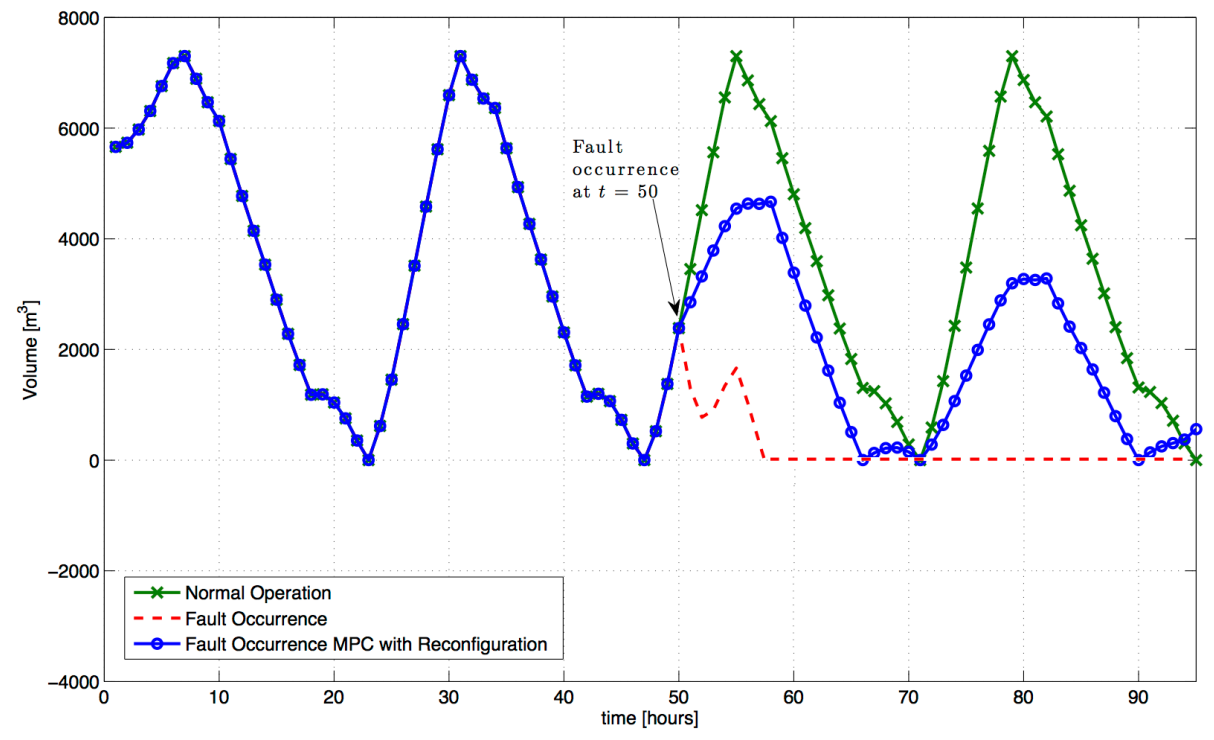

Figure 6: Volume evolution of Tank 33 with MPC using Reconfiguration

two actuators: 73 (iCornella130) and 108 (vTerMontcada). It can be seen that in a non-faulty situation, the volume of this tank presents a repetitive pattern (filling when pumping is cheaper and emptying otherwise) to satisfy the water demand. However, when a fault occurs (at $k=50$ hours), if the MPC controller is not reconfigured (labelled as fault occurrence in the plots), tank 73 volume drops to zero at $k=58$ hours and demand is not satisfied anymore (unfeasible solution). However, if the MPC controller is reconfigured by removing the faulty actuator 108 from the control model, the tank level is still able to supply the required demand. However, the tank volume decreases with time, indicating that the faulty actuator should be repaired. Figures 7 and 8, which depict the behaviour of actuators 108 and 73, show that actuator 73 starts to deliver more flow in an effort to compensate for the faulty actuator 108 that is removed.

Figures 9, 10 and 11 depict tank 33 volume and actuator 108 and 73 flows when the fault is accommodated by the MPC controller. The fault affecting actuator 108 reduces the operating range by $50 \%$. In this case, the faulty actuator is not removed from the control model of the MPC controller; rather, the operating limits of actuator 108 are updated according to the new operating range. Figure 9 shows how the volume behaviour of tank 33 in a non-fault situation and when using accommodation looks exactly the same; in contrast, when the controller is not accommodated, the volume tends to zero and demand is not satisfied.

From Figures 10 and 11, it can be seen that the MPC controller compensates for the reduction in the faulty actuator's operating range by increasing use of the non-faulty actuator, thereby compensating for the impact of the fault.

Although the proposed algorithm improves handling of the behaviour of the tank volume and actuator flows, it has computational and financial costs, as implementation of this feature increments computation time by $30 \mathrm{~s}(12 \%)$ and the cost overrun by 


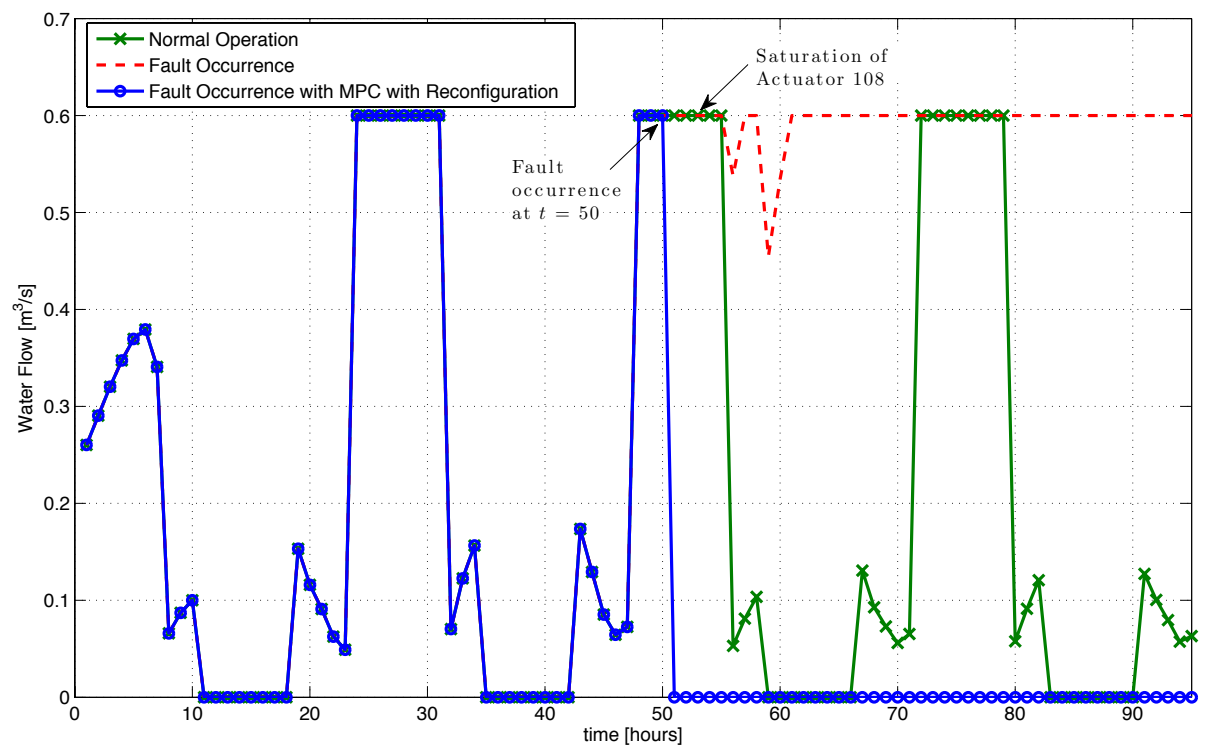

Figure 7: Water flow in Actuator 108 with MPC using Reconfiguration

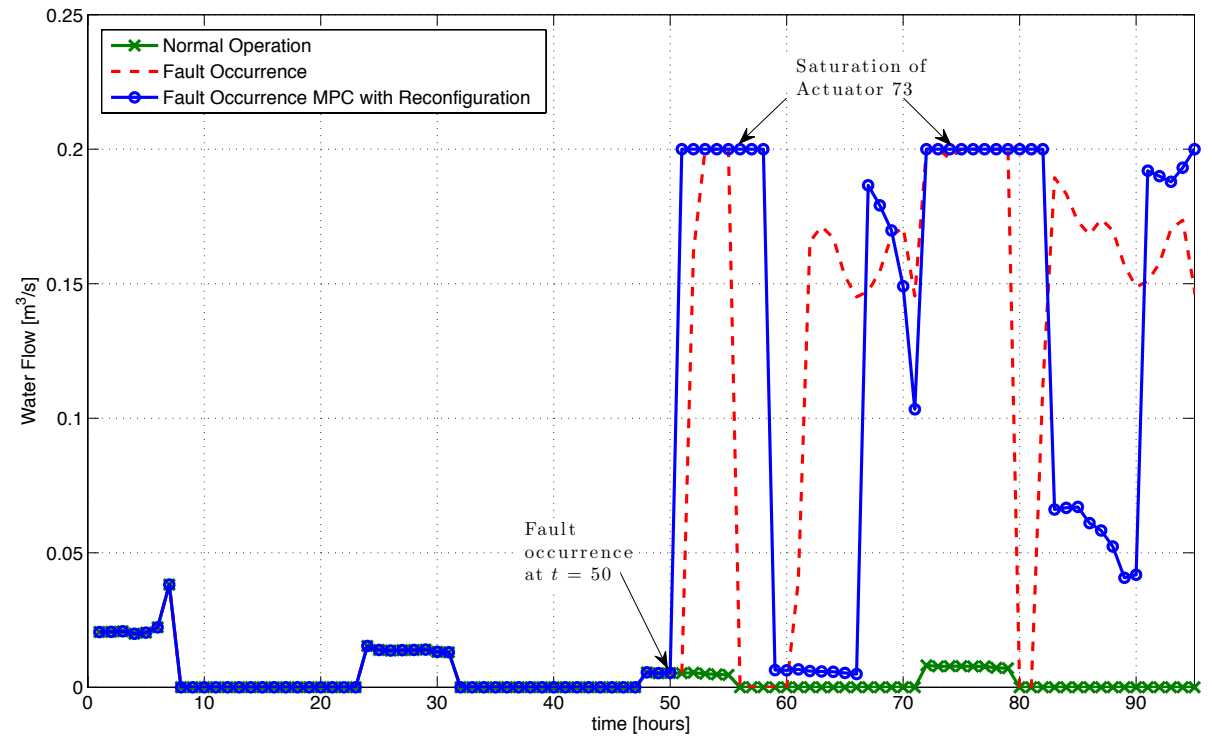

Figure 8: Water flow in Actuator 73 with MPC using Reconfiguration

around $9 \%$.

The reliability analysis also takes into account the results of the previous analysis. The reliability of the entire network considering proper operation is $90.74 \%$ success- 


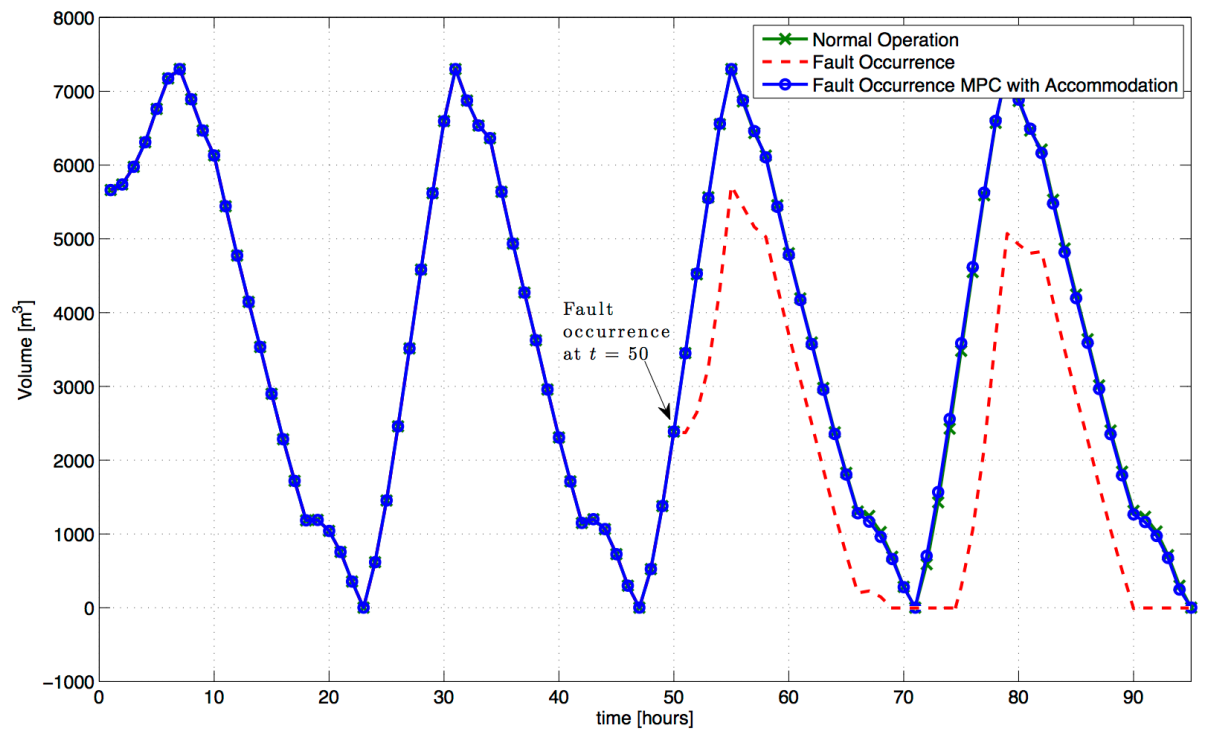

Figure 9: Evolution of volume in Tank 33 with MPC using Accommodation

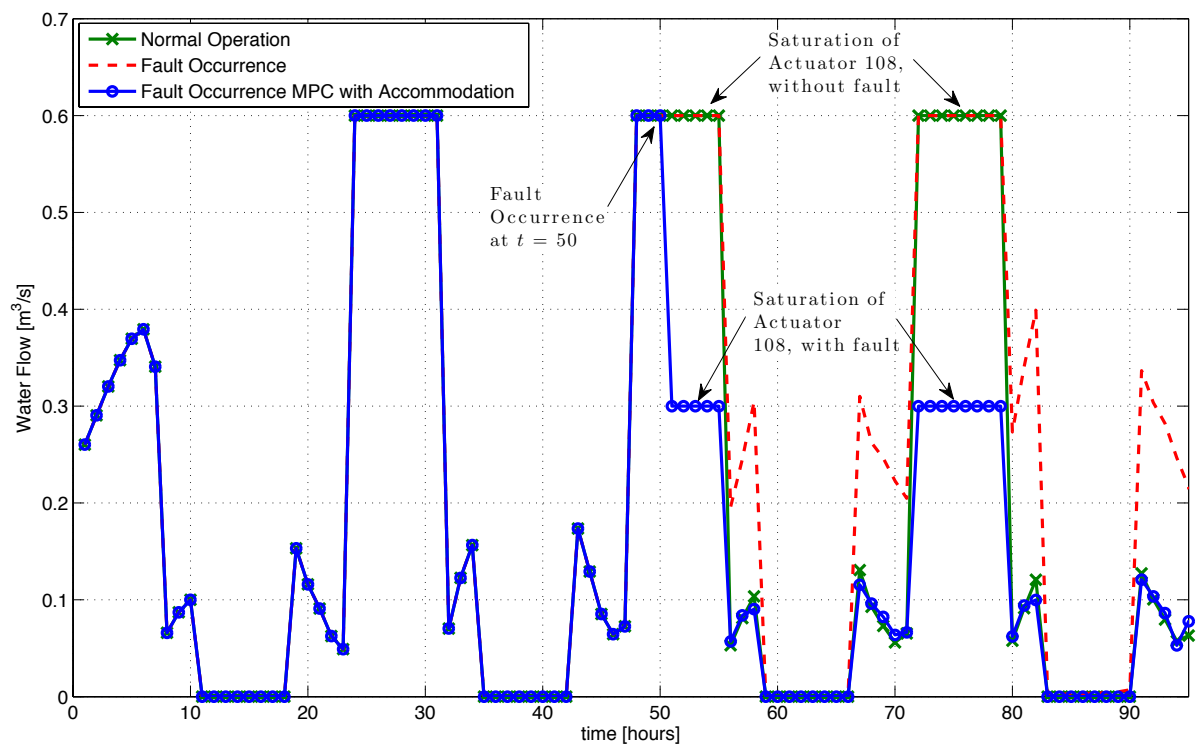

Figure 10: Water flow in Actuator 108 with MPC using Accommodation

ful in satisfying the desired property when the reliability of each component is calculated using (20) with $\lambda=0.0034$ (data obtained Ministry of Work and Social Affairs (2008)). The association between demand satisfaction and reduced reliability when a faulty component exists is shown in Table 4. 


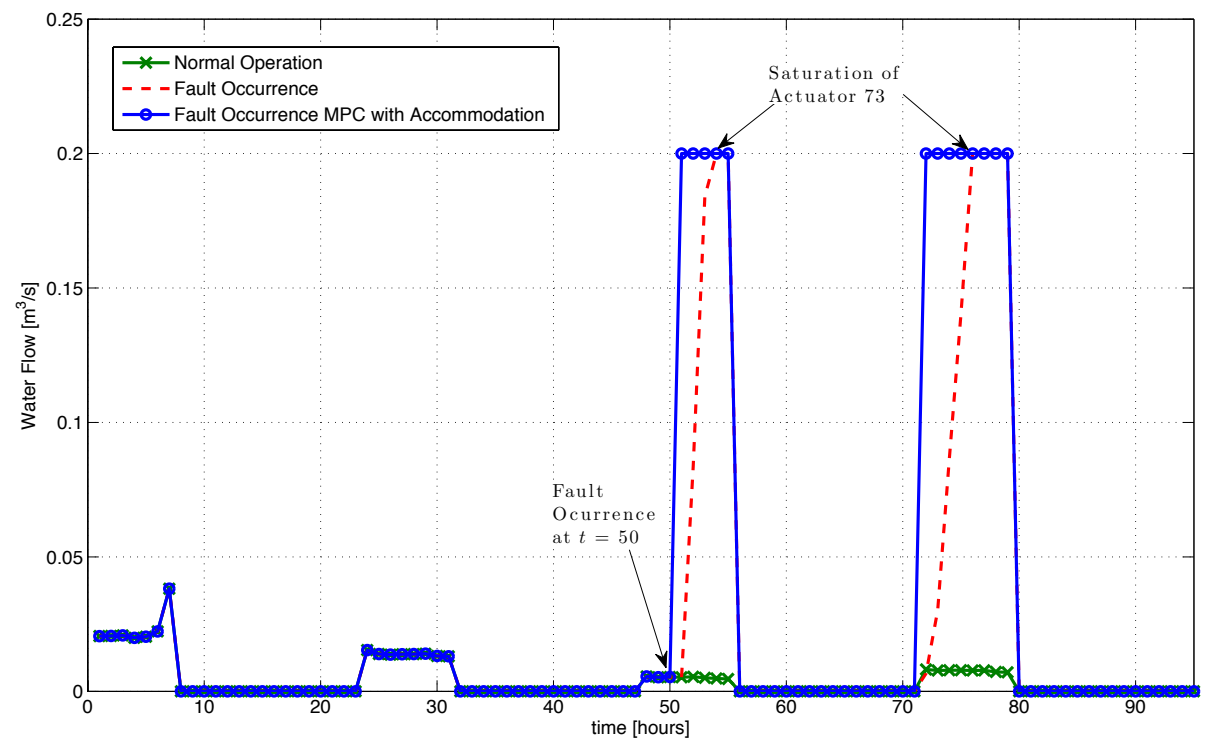

Figure 11: Water flow in Actuator 73 with MPC using Accommodation

As can be seen in Table 4, although most faults in actuators do not significantly affect reliability in satisfying demand, some completely override the satisfaction of the desired property. These actuators are critical actuators regarding reliability. The risk of having a malfunction in the system can be better understood when we compute the reliability of the entire network. Examples of critical actuators obtained from this study were actuators 102 and 103 since their malfunction led to a drop of 31.13\% in the reliability of the entire network.

The reliability analysis was applied to the network depicted in Figure 5. The reliability of satisfying Demand 56 decreased to $1.33 \%$ if actuators 52 and 58 had a fault, highlighting the importance of both these actuators for the operation of this smaller network, and decreased to $0 \%$ when actuators 43 and 54 were faulty, reaffirming the fact that these two actuators are critical. Otherwise, reliability remained the same. Regarding the entire DWTN, actuator 52 decreased reliability of satisfying the demands in the network by $21.71 \%$, denoting again that it is an important element in system interconnectivity.

Critical actuators 43 and 54, when they malfunction, reduced the reliability of the entire system towards zero; in contrast, the fact that other actuators did not affect reliability denote them to be redundant actuators.

Finally, the MPC redesign approach to preserve the network reliability has been applied to the entire DWTN using Algorithm 4. Figure 12 shows how the reliability of the network evolves in time when this algorithm is used. It can be observed that with the use of Algorithm 4, the reliability of the network degrades slowly compared to the case that the reliability is not considered in the MPC design. 
Table 4: Association between demand satisfaction and reliability

\begin{tabular}{cccc}
\hline $\begin{array}{c}\text { Demand } \\
\text { No. }\end{array}$ & $\begin{array}{c}\text { Percentage of } \\
\text { total demand [\%] }\end{array}$ & $\begin{array}{c}\text { Faulty } \\
\text { Components }\end{array}$ & $\begin{array}{c}R_{g}^{p} \text { in Faulty } \\
\text { conditions [\%] }\end{array}$ \\
\hline 69 & 9.1 & 128 & 0 \\
83 & 4.0069 & $82,88,90,104$ & 0 \\
70 & 3.2537 & 125 & 0 \\
70 & 3.2537 & 58 & 99.33 \\
70 & 3.2537 & $53,50,51$ & 99.99 \\
33 & 1.964 & 108 & 99.98 \\
58 & 1.9407 & 52,58 & 99.33 \\
56 & 1.6777 & 52,58 & 98.67 \\
64 & 1.4941 & 58,59 & 0 \\
\hline
\end{tabular}

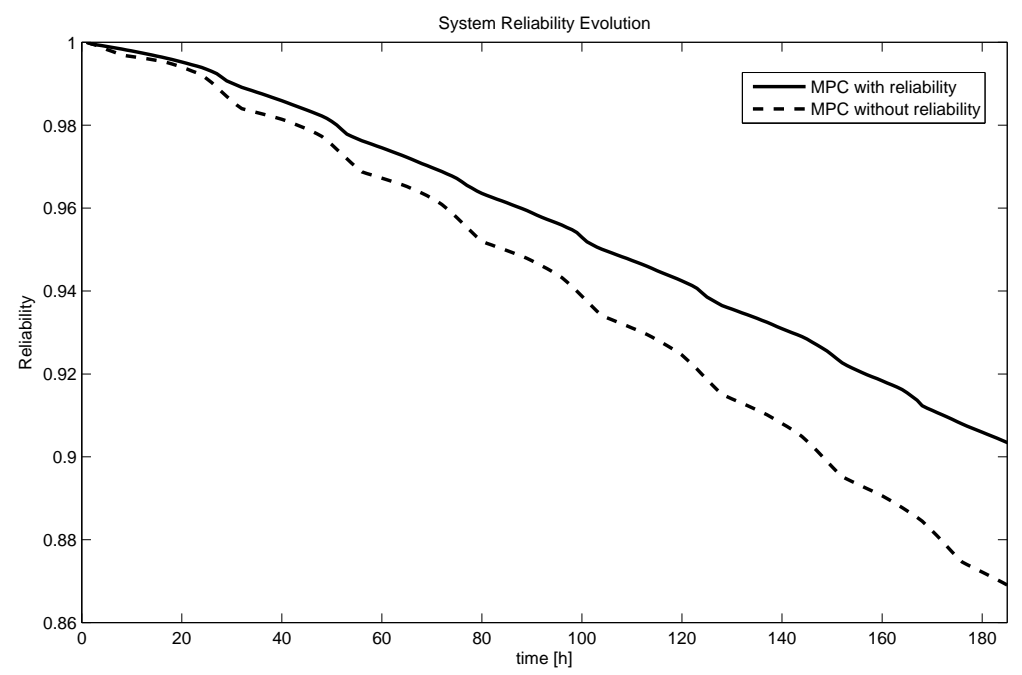

Figure 12: MPC redesign to preserve reliability 


\section{Conclusions}

This paper has proposed a reliable fault-tolerant model predictive control strategy for drinking water transport networks. The proposed approach combines structural, feasibility, performance and reliability analyses. After a fault, the predictive controller is redesigned to cope with the fault by considering either a reconfiguration or an accommodation strategy depending on available knowledge regarding the fault. Before starting to apply the fault-tolerant control strategy, whether the predictive controller will be able to continue operating after the fault appearance needs to be evaluated. This evaluation is performed by means of a structural analysis to determine post-fault loss of controllability, complemented with a feasibility analysis of the optimization problem related to the predictive control design, so as to consider the fault impact on actuator constraints. By evaluating the admissibility of different actuator-fault configurations, critical actuators regarding fault tolerance can be identified. The proposed approach also allows for a degradation analysis of the system in terms of performance and reliability. As a result of this analysis, the predictive controller design can be modified by adapting constraints such that the best achievable performance with some preestablished level of reliability is achieved. The proposed approach, successfully tested on the Barcelona water network, shows that relevant information can be extracted about critical actuators considered in the different analyses. Future research will investigate the impact of uncertainty on the analyses and on the design of the predictive controller including fault-tolerant capabilities.

\section{Appendix}

A. Graph reachability analysis

Definition A.1 (Path). Given a graph $G=(\mathcal{V}, \mathcal{E})$, a collection of vertices $v_{1}, v_{2}, \ldots, v_{k}$, together with the edges $\left(v_{1}, v_{2}\right),\left(v_{2}, v_{3}\right), \ldots,\left(v_{k-1}, v_{k}\right)$ placed in sequence, then the ordered set $\left(v_{1}, v_{2}\right),\left(v_{2}, v_{3}\right), \ldots,\left(v_{k-1}, v_{k}\right)$ is a path from $v_{1}$ to $v_{k}$.

Then, it is said that $v_{i}$ is reachable from $v_{j}$ if there is a path from $v_{j}$ to $v_{i}$.

Definition A.2 (Reachable set of a vertex). A reachable set $\mathcal{V}_{i}\left(v_{j}\right)$ of a vertex $v_{j}$ is a set $\mathcal{V}_{i}$ of vertices $v_{i}$ reachable from the vertex $v_{j} \in \mathcal{V}$.

Definition A.3 (Reachable set of a vertices set). A reachable set $\mathcal{V}_{i}\left(\mathcal{V}_{j}\right)$ is the set of vertices $v_{i}$ that are reachable from at least one vertex $v_{j} \in \mathcal{V}_{j}$.

Notice that $\mathcal{V}_{i}\left(\mathcal{V}_{j}\right)$ is the union of the sets $\mathcal{V}_{i}\left(v_{j}\right)$ for all $v_{j} \in \mathcal{V}_{j}$.

Definition A.4 (Input reachable system). A system with a digraph $G(\mathcal{V}, \mathcal{E})$, where $\mathcal{V}$ is the union of the set of states $\mathcal{X}$ and the set of inputs $\mathcal{U}$, is input reachable if the set of states $\mathcal{X}$ is a reachable set of the set of inputs $\mathcal{U}$.

From Definition A.4 it follows that if no input $u_{j}$ can reach a state $x_{i}$, either directly or through other states/inputs of the system, then there is no way of changing the system in order to satisfy the desired control objectives. 
In order to establish the input reachability of the system with digraph $G=(\mathcal{V}, \mathcal{E})$, the reachability matrix $\Gamma=\left(\Gamma_{i j}\right)$, defined as

$$
\Gamma_{i j}= \begin{cases}1 & \text { if } v_{i} \text { is reachable from } v_{j}, \\ 0 & \text { otherwise }\end{cases}
$$

must be computed. According to Šiljak (1991), a straightforward way to determine the reachability matrix $\Gamma$ is through the following expression:

$$
\Gamma_{i j}=\left\{\begin{array}{lll}
0 & \text { if } & q_{i j}=0 \\
1 & \text { if } \quad q_{i j} \neq 0
\end{array}\right.
$$

where $Q=\left(q_{i j}\right)=\Xi+\Xi^{2}+\ldots+\Xi^{s}$ and $\Xi$ is the interconnection matrix, defined as

$$
\Xi=\left[\begin{array}{ccc}
\bar{A} & \bar{B} & 0 \\
0 & 0 & 0 \\
\bar{C} & 0 & 0
\end{array}\right],
$$

and the submatrices $\bar{A}, \bar{B}, \bar{C}$ are Boolean representations of the original system matrices $A, B, C$ in state space.

Using (A.1), the reachability matrix is written as

$$
\Gamma=\left[\begin{array}{ccc}
F & E & 0 \\
0 & 0 & 0 \\
H & \theta & 0
\end{array}\right] .
$$

For the detailed procedure to determine the reachability matrix (A.4), i.e., the binary matrices $F, E, H$ and $\theta$, the reader is referred to Šiljak (1991). Additionally, the following theorems should be recalled.

Theorem 1 (Šiljak (1991)). A system given by (1) is input reachable if and only if computing the reachability matrix (A.4), the binary matrix $E$ has no zero rows, and it is output reachable if and only if the binary matrix $H$ has no zero columns.

Theorem 2 (Šiljak (1991)). A system given by (1) is structurally controllable if and only if it is input reachable according to Theorem 1 and the structural rank ${ }^{8}$ equals to $n_{x}$, i.e., s-rank $([A B])=n_{x}$.

\section{B. Constraint satisfaction problem}

A constraint satisfaction problem (CSP) on sets can be formulated as a 3-tuple $\mathcal{H}=(\mathcal{W}, \mathcal{D}, \mathcal{Z})($ Jaulin et al., 2001), where

- $\mathcal{W}=\left\{w_{1}, \ldots, w_{n}\right\}$ is a finite set of variables;

\footnotetext{
${ }^{8}$ The structural rank of a matrix is the maximum rank of all numerical matrices with the same non-zero pattern. It can be easily computed in MATLAB using the srank command.
} 
- $\mathcal{D}=\left\{\mathcal{D}_{1}, \ldots, \mathcal{D}_{n}\right\}$ is the set of their domains (where, in this paper, $\mathcal{D}_{i} \subseteq \mathbb{R}, i=$ $1, \ldots, n)$

- $\mathcal{Z}=\left\{z_{1}, \ldots, z_{n}\right\}$ is a finite set of constraints relating variables of $\mathcal{W}$.

A point solution of $\mathcal{H}$ is a n-tuple denoted by $\left(\tilde{w}_{1}, \ldots, \tilde{w}_{n}\right) \in \mathcal{D}$ such that all constraints $\mathcal{Z}$ are satisfied. The set of all point solutions of $\mathcal{H}$ is denoted by $\mathcal{S}_{\mathcal{H}}$. This set is called the global solution set. The variable $w_{i} \in \mathcal{W}$ is consistent in $\mathcal{H}$ if and only if

$$
\forall \tilde{w}_{i} \in \mathcal{D}_{i} \exists\left(\tilde{w}_{1} \in, \mathcal{D}_{1} \ldots, \tilde{w}_{n} \in \mathcal{D}_{n}\right) \mid\left(\tilde{w}_{1}, \ldots, \tilde{w}_{n}\right) \in \mathcal{S}_{\mathcal{H}},
$$

with $i=1 \ldots n$. The solution of a CSP is said to be globally consistent, if and only if every variable is consistent considering the whole set of constraints. A variable is locally consistent if and only if it is consistent with respect to a group of constraints. Thus, the solution of a CSP is said to be locally consistent if all variables are locally consistent.

\section{Acknowledgements}

This work has been partially funded by the Spanish Ministry of Science and Technology through the Project ECOCIS (Ref. DPI2013-48243-C2-1-R) and Project HARCRICS (Ref. DPI2014-58104-R).

\section{References}

Blanke, M., Kinnaert, M., Lunze, J., \& Staroswiecki, M. (2016). Diagnosis and FaultTolerant Control. (3rd ed.). Berlin, Heidelberg: Springer-Verlag.

Bondy, J., \& Murty, U. (1982). Graph Theory with Applications. Great Britain: MacMillan Press.

Brdys, M., \& Ulanicki, B. (1994). Operational Control of Water Systems: Structures, Algorithms and Applications. Prentice Hall International.

Camacho, E. F., Alamo, T., \& Muñoz de la Peña, D. (2010). Fault-tolerant model predictive control. In IEEE Conference on Emerging Technologies and Factory Automation (ETFA'2010) (pp. 1-8).

Ellis, M., Durand, H., \& Christofides, P. D. (2014). A tutorial review of economic model predictive control methods. Journal of Process Control, 24, $1156-1178$.

Gertsbakh, I. B. (2000). Reliability Theory with Application to Preventive Maintenance. Springer-Verlag.

Guenab, F., Weber, P., Theilliol, D., \& Zhang, Y. M. (2011). Design of a fault tolerant control system incorporating reliability analysis and dynamic behaviour constraints. International Journal of Systems Science, 42, 219-233. 
Jaulin, L., Kieffer, M., Braems, I., \& Walter, E. (2001). Guaranteed nonlinear estimation using constraint propagation on sets. International Journal of Control, 74, 1772-1782.

Limon, D., Pereira, M., Muñoz de la Peña, D., Alamo, T., \& Grosso, J. (2014). Singlelayer economic model predictive control for periodic operation. Journal of Process Control, 8, 1207-1224.

Maciejowski, J. (2002). Predictive Control with Constraints. Great Britain: Prentice Hall.

Ministry of Work and Social Affairs (2008). NTP 417: Quantitative risk analysis Reliability of components and implications in preventive maintenance (in Spanish only). Technical Report Spain.

Noura, H., Theilliol, D., Ponsart, J., \& Chamssedine, A. (2009). Fault tolerant control systems: Design and practical application. Springer Verlag.

Ocampo-Martinez, C., \& Puig, V. (2009). Fault-tolerant model predictive control in the hybrid systems framework: Application to sewer networks. International Journal of Adaptive Control and Signal Processing, 23, 757-787.

Ocampo-Martinez, C., Puig, V., Cembrano, G., \& Quevedo, J. (2013). Application of predictive control strategies to the management of complex networks in the urban water cycle. Control Systems, IEEE, 33, 15-41.

Ostfeld, A. (2001). Reliability analysis of regional water distribution systems. Urban Water, 3, 253-260.

Pascual, J., Romera, J., Puig, V., Cembrano, G., Creus, R., \& Minoves, M. (2013). Operational predictive optimal control of Barcelona water transport network. Control Engineering Practice, 21, 1020-1034.

Prodan, I., Zico, E., \& Stoican, F. (2015). Fault tolerant predictive control design for reliable microgrid energy management under uncertainties. Energy, 91, 20 - 34.

Raimondo, D. M., Marseglia, G. R., Braatz, R., \& Scott, J. K. (2013). Fault-tolerant model predictive control with active fault isolation. In 2 nd Conference on Control and Fault-Tolerant Systems (SysTol) (pp. 444-449). Nice, France.

Rawlings, J. B., Angeli, D., \& Bates, C. N. (2012). Fundamentals of economic model predictive control. In 51st IEEE Conference on Decision and Control. Maui, Hawaii, USA.

Robles, D., Puig, V., Ocampo-Martinez, C., \& Garza, L. E. (2012). Methodology for actuator fault tolerance evaluation of linear constrained MPC: Application to the Barcelona water network. In 20th Mediterranean Conference on Control Automation (MED) (pp. 518-523). 
Salazar, J., Weber, P., Sarrate, R., Theilliol, D., \& Nejari, F. (2015). MPC design based on a DBN reliability model: application to drinking water networks. In 9th IFAC Symposium on Fault Detection, Supervision and Safety of Technical Processes (pp. 688-693). Paris, France.

Sanchez, H., Escobet, T., Puig, V., \& P. F, O. (2015). Health-aware model predictive control of wind turbines using fatigue prognosis. In 9th IFAC Symposium on Fault Detection, Supervision and Safety of Technical Processes (pp. 1363-1368). Paris, France.

Šiljak, D. (1991). Decentralized control of complex systems. Academic Press.

Staroswiecki, M., \& Berdjag, D. (2010). A general fault tolerant linear quadratic control strategy under actuator outages. International Journal of Systems Science, 41, 971-985.

Staroswiecki, M., Commault, C., \& Dion, J. (2012). Fault tolerance evaluation based on the lattice of system configurations. International Journal of Adaptive Control and Signal Processing, 26, 54-72.

Torii, A., \& Lopez, R. (2012). Reliability analysis of water distribution networks using the adaptive response surface approach. Journal of Hydraulic Engineering, 138, 227-236.

Wang, Y., Ocampo-Martinez, C., \& Puig, V. (2015). Robust model predictive control based on Gaussian processes: Application to drinking water networks. In European Control Conference. Linz (Austria).

Weber, P., Simon, C., Theilliol, D., \& Puig, V. (2012). Fault-tolerant control design for over-actuated system conditioned by reliability: A drinking water network application. In 8th IFAC Symposium on Fault Detection, Supervision and Safety for Technical Processes (Safeprocess12). Mexico City, Mexico.

Yang, X., \& Maciejowski, J. (2012). Fault-tolerant model predictive control of a wind turbine benchmark. In 8th IFAC Symposium on Fault Detection, Supervision and Safety of Technical Processes (pp. 337-342). Mexico City, Mexico.

Yetendje, A., Seron, M., \& Doná, J. D. (2012). Fault-tolerant model predictive control in the hybrid systems framework: Application to sewer networks. International Journal of Applied Mathematics and Computer Science, 22, 211-223.

Zhang, Y., \& Jiang, J. (2008). Bibliographical review on reconfigurable fault-tolerant control systems. Annual Reviews in Control, 32, 229 - 252. 